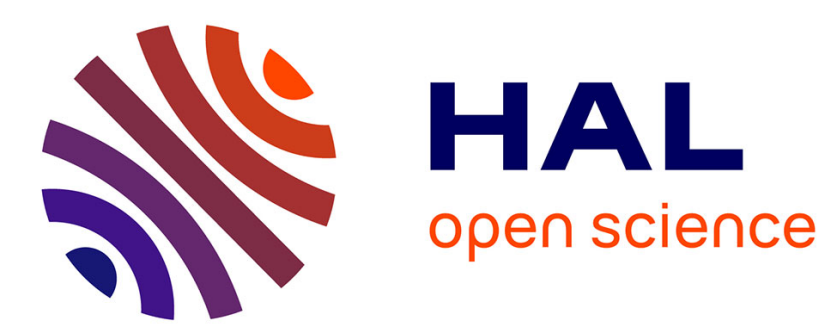

\title{
A Wildly Flickering Jet in the Black Hole X-Ray Binary MAXI J1535-571
}

Maria Cristina Baglio, David M. Russell, Piergiorgio Casella, Hind Al Noori, Aisha Al Yazeedi, Tomaso Belloni, David A.H. Buckley, Marion Cadolle Bel, Chiara Ceccobello, Stephane Corbel, et al.

\section{To cite this version:}

Maria Cristina Baglio, David M. Russell, Piergiorgio Casella, Hind Al Noori, Aisha Al Yazeedi, et al.. A Wildly Flickering Jet in the Black Hole X-Ray Binary MAXI J1535-571. Astrophys.J., 2018, 867 (2), pp.114. 10.3847/1538-4357/aae532 . hal-01871900

\section{HAL Id: hal-01871900 https://hal.science/hal-01871900}

Submitted on 12 Jun 2020

HAL is a multi-disciplinary open access archive for the deposit and dissemination of scientific research documents, whether they are published or not. The documents may come from teaching and research institutions in France or abroad, or from public or private research centers.
L'archive ouverte pluridisciplinaire HAL, est destinée au dépôt et à la diffusion de documents scientifiques de niveau recherche, publiés ou non, émanant des établissements d'enseignement et de recherche français ou étrangers, des laboratoires publics ou privés. 


\title{
A Wildly Flickering Jet in the Black Hole X-Ray Binary MAXI J1535-571
}

Maria Cristina Baglio ${ }^{1,2}$ (1) , David M. Russell ${ }^{1}$ (1), Piergiorgio Casella ${ }^{3}$, Hind Al Noori ${ }^{1}$, Aisha Al Yazeedi ${ }^{1}$, Tomaso Belloni ${ }^{2}$ (1), David A. H. Buckley ${ }^{4}$, Marion Cadolle $\mathrm{Bel}^{5}$, Chiara Ceccobello ${ }^{6}$, Stephane Corbel ${ }^{7,8}$, Francesco Coti Zelati ${ }^{2,9,10}{ }_{(10}$, Maria Díaz Trigo ${ }^{11}$ (i), Rob P. Fender ${ }^{12}$, Elena Gallo ${ }^{13}$, Poshak Gandhi ${ }^{14}$ (1) , Jeroen Homan ${ }^{15,16}$ (D), Karri I. I. Koljonen ${ }^{17,18}$ (D), Fraser Lewis ${ }^{19,20}$, Thomas J. Maccarone ${ }^{21}$, Julien Malzac ${ }^{22}$, Sera Markoff ${ }^{23}$ (1), James C. A. Miller-Jones ${ }^{24}$ (1) , Kieran O’Brien ${ }^{25}$, Thomas D. Russell $^{23}$ (10), Payaswini Saikia ${ }^{1}$, Tariq Shahbaz ${ }^{26,27}$, Greg R. Sivakoff ${ }^{28}$ (D), Roberto Soria ${ }^{24,29,30}$, Vincenzo Testa ${ }^{3}$ (D), Alexandra J. Tetarenko 28,31 (D), Mario E. van den Ancker $^{11}$ (i), and Federico M. Vincentelli ${ }^{2,3,32}$

${ }^{1}$ New York University Abu Dhabi, PO Box 129188, Abu Dhabi, UAE; mcb19@nyu.edu

2 INAF, Osservatorio Astronomico di Brera, Via E. Bianchi 46, I-23807 Merate (LC), Italy

3 INAF, Osservatorio Astronomico di Roma, Via Frascati 33, I-00040, Monteporzio Catone (RM), Italy

${ }^{4}$ South African Astronomical Observatory, P.O. Box 9, Observatory 7935, Cape Town, South Africa ${ }^{5}$ SIXT Leasing SE, Zugspitzstr. 1, D-82049 Pullach (Munich), Germany

${ }^{6}$ Department of Space, Earth and Environment, Chalmers University of Technology, Onsala Space Observatory, SE-439 92 Onsala, Sweden ${ }_{7}^{7}$ Laboratoire AIM (CEA/IRFU-CNRS/INSU-Université Paris Diderot), CEA DSM/IRFU/SAp, F-91191 Gif-sur-Yvette, France

${ }^{8}$ Station de Radioastronomie de Nançay, Observatoire de Paris, PSL Research University, CNRS, Univ. Orléans, F-18330 Nançay, France

${ }^{9}$ Institute of Space Sciences (ICE, CSIC), Campus UAB, Carrer de Can Magrans s/n, E-08193 Barcelona, Spain

${ }^{10}$ Institut d'Estudis Espacials de Catalunya (IEEC), E-08034 Barcelona, Spain

${ }^{11}$ ESO, Karl-Schwarzschild-Strasse 2, D-85748 Garching bei München, Germany

12 Astrophysics, Department of Physics, University of Oxford, Keble Road, Oxford OX1 3RH, UK

13 Department of Astronomy, University of Michigan, 1085 S University, Ann Arbor, MI 48109, USA

${ }^{14}$ University of Southampton, Department of Physics \& Astronomy, Southampton, SO17 1BJ, UK

${ }^{16}$ SRON, Netherlands Institute for Space Research, Sorbonnelaan 2, 3584 CA Utrecht, The Netherlands

${ }^{17}$ Finnish Centre for Astronomy with ESO (FINCA), University of Turku, Väisäläntie 20, FI-21500 Piikkiö, Finland

${ }^{18}$ Aalto University Metsähovi Radio Observatory, PO Box 13000, FI-00076 Aalto, Finland

${ }^{19}$ Faulkes Telescope Project, School of Physics and Astronomy, Cardiff University, The Parade, Cardiff CF24 3AA, UK

${ }^{20}$ Astrophysics Research Institute, Liverpool John Moores University, 146 Brownlow Hill, Liverpool L3 5RF, UK

${ }^{21}$ Department of Physics and Astronomy, Texas Tech University, Box 41051, Lubbock, TX 79409-1051, USA

${ }^{22}$ IRAP, Universit de Toulouse, CNRS, UPS, CNES, Toulouse, France

23 Anton Pannekoek Institute for Astronomy, University of Amsterdam, 1098 XH Amsterdam, The Netherlands

24 International Centre for Radio Astronomy Research, Curtin University, GPO Box U1987, Perth, Western Australia 6845, Australia ${ }_{25}$ Department of Physics, Durham University, South Road, Durham, DH1 3LE, UK

${ }^{26}$ Instituto de Astrofísica de Canarias (IAC), E-38200 La Laguna, Tenerife, Spain

27 Departamento de Astrofísica, Universidad de La Laguna (ULL), E-38206 La Laguna, Tenerife, Spain

${ }^{28}$ Department of Physics, University of Alberta, CCIS 4-181, Edmonton, AB T6G 2E1, Canada

${ }^{29}$ College of Astronomy and Space Sciences, University of Chinese Academy of Sciences, Beijing 100049, People's Republic of China

${ }^{30}$ Sydney Institute for Astronomy, School of Physics A28, The University of Sydney, Sydney, NSW 2006, Australia

${ }^{31}$ East Asian Observatory, 660 N. A'ohoku Place, University Park, Hilo, Hawaii 96720, USA

32 DiSAT, Università degli Studi dellInsubria, Via Valleggio 11, I-22100 Como, Italy

Received 2018 July 16; revised 2018 September 20; accepted 2018 September 28; published 2018 November 6

\begin{abstract}
We report on the results of optical, near-infrared (NIR), and mid-infrared observations of the black hole X-ray binary candidate (BHB) MAXI J1535-571 during its 2017/2018 outburst. During the first part of the outburst (MJD 58004-58012), the source shows an optical-NIR spectrum that is consistent with an optically thin synchrotron power law from a jet. After MJD 58015, however, the source faded considerably, the drop in flux being much more evident at lower frequencies. Before the fading, we measure a dereddened flux density of $\gtrsim 100 \mathrm{mJy}$ in the mid-infrared, making MAXI J1535-571 one of the brightest mid-infrared BHBs known so far. A significant softening of the X-ray spectrum is evident contemporaneous with the infrared fade. We interpret it as being due to the suppression of the jet emission, similar to the accretion-ejection coupling seen in other BHBs. However, MAXI J1535-571 did not transition smoothly to the soft state, instead showing X-ray hardness deviations associated with infrared flaring. We also present the first mid-IR variability study of a BHB on minute timescales, with a fractional rms variability of the light curves of $\sim 15 \%-$ $22 \%$, which is similar to that expected from the internal shock jet model, and much higher than the optical fractional rms $(\lesssim 7 \%)$. These results represent an excellent case of multiwavelength jet spectral timing and demonstrate how rich, multiwavelength time-resolved data of X-ray binaries over accretion state transitions can help in refining models of the disk-jet connection and jet launching in these systems.
\end{abstract}

Key words: accretion, accretion disks - black hole physics - ISM: jets and outflows - X-rays: binaries

\section{Introduction}

Low-mass X-ray binaries (LMXBs) are systems that typically host an evolved/main-sequence star and a compact object, either a neutron star (NS) or a black hole (BH). The companion star of these systems transfers mass and angular momentum toward the compact object via Roche lobe overflow and the formation of an accretion disk. LMXBs can be transient, which means that they alternate between outbursts, typically lasting weeks to months, with X-ray luminosities reaching $10^{36}-10^{38} \mathrm{erg} \mathrm{s}^{-1}$ and high accretion levels, and 
longer (years to decades) periods of quiescence, with a typical drop in the X-ray luminosity by 5-6 orders of magnitude.

A coupling between accretion and ejection of matter is thought to exist for LMXBs (Merloni et al. 2003; Falcke et al. 2004; Plotkin et al. 2013). These ejections are mainly seen in the form of collimated and compact jets coming from a region that is very near the central compact object. The production of relativistic jets in black hole X-ray binaries (BHBs) is a matter of extensive study in the field of accreting systems. The magnetic field in the inner part of the accretion flow is thought to be responsible for particle acceleration and jet launching. In the case of jet emission, a flat radio spectrum is typically observed when the system is in its hard X-ray state (Fender 2001; Fender et al. 2004; Corbel et al. 2004). This flat or inverted ( $\alpha \sim 0-0.5$, with $\left.F_{\nu} \propto \nu^{\alpha}\right)$ spectrum is one of the principal signatures of the presence of a collimated jet in BHBs. It typically extends at least to the millimeter regime and is normally explained as being due to the superposition of selfabsorbed synchrotron emission components originating at different distances from the central BH. At higher frequencies, often starting from the mid-infrared (MIR) or near-infrared (NIR) bands, optically thin synchrotron emission is observed, resulting in a power law with index $-1 \leqslant \alpha \leqslant-0.5$ (Russell et al. 2013b). The transition between the optically thick and thin parts of the spectrum happens at the so-called jet break frequency, $\nu_{\mathrm{b}}$, which usually falls in the MIR to NIR (Russell et al. 2013b). In the soft X-ray state instead, jets appear to be quenched at all frequencies (see, e.g., Russell et al. 2011) in favor of disk winds (Ponti et al. 2012), probably due to the suppression of the magnetic field caused by the geometrically thin accretion disk that resides close to the $\mathrm{BH}$ (see, e.g., Meier 2001). Alternatively, in line with the internal shock model for BHB jets by Malzac (2013), during the soft state, the jet might still be present but is dark because of the lack of variability in the disk (Drappeau et al. 2017). In some cases, the jet break frequency has been found to move from infrared to radio frequencies as the X-ray spectrum of the source softens, and then come back again to the infrared band at the end of the outburst, when the spectrum gets harder (Russell et al. 2013a, 2014; Diaz Trigo et al. 2018). This behavior suggests that the structure of the accretion flow determines the position of the break frequency of the jet.

The size of the jet-emitting region is thought to scale inversely with the frequency (Blandford \& Königl 1979); in this way, $\nu_{\mathrm{b}}$ would give information on the size of the base of the jet, that is, where the particle acceleration starts (Heinz \& Sunyaev 2003; Chaty et al. 2011; Ceccobello et al. 2018). Moreover, the detection of the jet break frequency is fundamental also to estimating the magnetic field strength and the total power of the jet and its radiative luminosity, and therefore to shed light on the jet formation process and the inflow/outflow connection in X-ray binaries (Russell et al. 2014). The break has been detected in the case of the BH candidates GX 339-4 (Corbel \& Fender 2002; Gandhi et al. 2011; Corbel et al. 2013), XTE J1118+480 (Hynes et al. 2006), XTE J1550-564 (Chaty et al. 2011), V404 Cyg (Russell et al. 2013b; Tetarenko et al. 2018), MAXI J1836-194 (Russell et al. 2013a, 2014), Cyg X-1 (Rahoui et al. 2011), and, in quiescence, Swift J1357.2-0933 and A0620-00 (Plotkin et al. 2016; Dinçer et al. 2018; Russell et al. 2018), and in the NS systems 4U 0614+091, 4U 1728-34, and
Aql X-1 (Migliari et al. 2010; Díaz Trigo et al. 2017; Diaz Trigo et al. 2018).

\section{MAXI J1535-571}

The BHB MAXI J1535-571 (hereafter J1535) was first detected by the MAXI/GSC nova alert system as a bright, hard X-ray transient on 2017 September 2 (Negoro et al. 2017b). Independently, the Swift/Burst Alert Telescope (Swift/BAT) instrument triggered on an outburst from a possible gamma-ray burst (Kennea et al. 2017), coming from the same position, which led to the conclusion that they were observing the same event. The spectrum of the source was well fit by an absorbed power law with a hydrogen column density $N_{\mathrm{H}}=(3.6 \pm 0.2) \times 10^{22} \mathrm{~cm}^{-2}$ and a photon index of $1.53 \pm 0.07$ (Kennea et al. 2017).

The optical counterpart was first detected on 2017 September 3 using the $0.61 \mathrm{~m} \mathrm{B \& C}$ Telescope operated by the University of Canterbury at Mt. John Observatory, located in New Zealand, which revealed a $i^{\prime}=21.8 \pm 0.2$ mag source inside the Swift/X-ray Telescope (Swift/XRT) error circle (Scaringi 2017). The target was not detected at higher frequencies (i.e., $g^{\prime}$ and $r^{\prime}$ bands), not surprisingly, because of the high extinction at that location in the Galactic Plane.

In the $2-20 \mathrm{keV}$ range, the X-ray flux increased linearly following the first detection (Negoro et al. 2017a). From further MAXI/GSC observations reported in Negoro et al. (2017a), the unabsorbed 1-60 keV flux $\left(3.1 \times 10^{-8} \mathrm{erg}^{-1} \mathrm{~s}^{-1} \mathrm{~cm}^{-2}\right)$ corresponded to a luminosity of $2.4 \times 10^{38} \mathrm{erg} \mathrm{s}^{-1}$ assuming a $8 \mathrm{kpc}$ distance, which exceeds the Eddington luminosity of a canonical $1.4 M_{\odot}$ NS by a factor of $\sim 1.4$. Moreover, rapid $\mathrm{X}$-ray variability without clear periodicity is observed in the GSC light curve. Due to these observed properties, the authors suggested that the source could be a hard-state, low-mass X-ray binary hosting a $\mathrm{BH}$.

Observations with the Australia Telescope Compact Array (ATCA) were performed (Russell et al. 2017b) on 2017 September 5 at 5.5 and $9 \mathrm{GHz}$. The authors detected a radio source at a position consistent with the X-ray one, with a radio spectral index of $\alpha=0.09 \pm 0.03$, which is consistent with emission from a compact radio jet. They also estimated the distance of the target to be about $6.5 \mathrm{kpc}$, assuming the source to be as close to the Galactic center as possible along its line of sight. The observed radio emission is well above the expected radio luminosity of an NS low-mass X-ray binary at the observed X-ray luminosity, whereas it is comparable to those of typical BHBs, indicating that $\mathrm{J} 1535$ is indeed a strong BHB candidate. Successively, the spectral and timing analysis of archival Swift (BAT and XRT) and MAXI (GSC) data reported in Shang et al. (2018) led to an estimate of the probable $\mathrm{BH}$ mass of $8.78_{-1.05}^{+1.22} M_{\odot}$.

The NIR counterpart was detected with the SMARTS $1.3 \mathrm{~m}$ telescope at CTIO as a $J=14.88, H=13.11$ mag source on 2017 September 5 (Dincer 2017).

The MAXI/GSC and Swift monitoring of the outburst revealed a linearly increasing trend of the $\mathrm{X}$-ray luminosity with time, the $2-20 \mathrm{keV}$ flux reaching $5.3 \times 10^{-8} \mathrm{erg} \mathrm{cm}^{-2} \mathrm{~s}^{-1}$ on September 10. After this date, while the MAXI 2-4 keV and the XRT count rate continued to increase, the MAXI 10-20 keV and the BAT count rates started to decline, while the X-ray photon index became softer, indicating a possible hard-soft transition of the source (Kennea 2017; Nakahira et al. 2017; Palmer et al. 2017). 
On 2017 September 11, millimeter observations with the Atacama Large Millimeter/Sub-millimeter array (ALMA) were performed. ALMA observations taken at 97, 140, and $230 \mathrm{GHz}$ marked $\mathrm{J} 1535$ as one of the brightest X-ray binaries ever detected at submillimeter wavelengths (Tetarenko et al. 2017). ATCA observations performed on September 12 confirmed the brightening of the source in the radio band, both at 17 and $19 \mathrm{GHz}$ (Tetarenko et al. 2017). The authors linked these radio detections to synchrotron emission arising from a compact jet, with a spectrum consistent with a single power law extending from the radio up to $\sim 140 \mathrm{GHz}$, with the break residing above that.

A softening of the X-ray spectrum on 2017 September 19 (MJD 58015) was first reported by Shidatsu et al. (2017b), suggesting that the source entered the so-called soft-intermediate state (Tao et al. 2018). A further brightening in the radio was then detected with ATCA on October 25 (Russell et al. 2017a), with a radio spectrum consistent with a single power law (spectral index of $\sim 0.09 \pm 0.01$ ), again indicative of synchrotron emission from a compact jet. This suggested that the source may have been transitioning back toward the hard state.

Shidatsu et al. (2017a) reported on a steady spectral softening of the source after 2017 October 25, reaching a hardness ratio of the $M A X I /$ GSC fluxes $(6-20 \mathrm{keV} / 2-6 \mathrm{keV})$ of $\sim 0.03$ in the last 10 days until November 27. This is the lowest hardness ratio reached by $\mathrm{J} 1535$ during the outburst and is significantly lower than the one measured soon before the temporary hardening that occurred at the end of October $(\sim 0.1)$, thus indicating that the source finally reached the soft state, where it remained for several months (see Section 5 and the work by Tao et al. 2018 for a detailed description of the evolution of the X-ray outburst of J1535).

Here, we present optical, NIR, and MIR observations of J1535 during the outburst rise and period of spectral transitions. We use the evolution of the emission properties to probe the disk-jet connection in this source. We also present the first MIR rapid (minute timescale) variations reported in a transient BHB.

\section{Observations}

\subsection{Optical and NIR Monitoring}

J1535 was extensively monitored during its 2017 outburst using the $2 \mathrm{~m}$ Faulkes Telescope South (FTS) located at Siding Spring (Australia), the Las Cumbres Observatory (LCO) robotic network of $1 \mathrm{~m}$ telescopes at Cerro Tololo InterAmerican Observatory in Chile (LSC), the South African Astronomical Observatory at Sutherland in South Africa (CPT) and Siding Spring (COJ), and the $1 \mathrm{~m}$ ESO Rapid Eye Mount telescope (REM) in La Silla (Chile).

\subsubsection{Faulkes/LCO Observations}

We observed J1535 with FTS and the $1 \mathrm{~m}$ LCO network on 20 nights in September and October 2017, from the first optical detection until the source was no longer visible from the ground. These observations are part of an ongoing monitoring campaign of $\sim 40$ LMXBs (Lewis et al. 2008). Imaging data were taken in the SDSS $g^{\prime} r^{\prime} i^{\prime}$ and Pan-STARRS $y$-band filters (4770-10040 $\AA$ ). We detected the source (with errors of $\leqslant 0.4 \mathrm{mag}$ ) in zero, one, 12 , and 11 images in the $g^{\prime}, r^{\prime}, i^{\prime}$, and $y$ bands, respectively. As for the REM observations, the nondetections at shorter wavelengths are very likely due to the
Table 1

Detailed Log of the Faulkes/LCO Observations Performed between 2017 September 4 and October 13

\begin{tabular}{lcccc}
\hline \hline Epoch & MJD & Telescope & Filters & $\begin{array}{c}\text { Exposures } \\
(\mathrm{s})\end{array}$ \\
\hline 2017 Sep 04 & 58000.37035 & FTS & $r^{\prime}$ & $3 \times 300$ \\
2017 Sep 06 & 58002.42514 & FTS & $i^{\prime}$ & 200 \\
2017 Sep 06 & 58002.78674 & $1 \mathrm{~m}-\mathrm{S}$ & $g^{\prime} i^{\prime}$ & 300,200 \\
2017 Sep 07 & 58003.42129 & $1 \mathrm{~m}-\mathrm{A}$ & $g^{\prime} i^{\prime}$ & 300,200 \\
2017 Sep 07 & 58003.78054 & $1 \mathrm{~m}-\mathrm{S}$ & $g^{\prime} i^{\prime}$ & 300,200 \\
2017 Sep 07 & 58003.78771 & $1 \mathrm{~m}-\mathrm{S}$ & $r^{\prime} y$ & 200,200 \\
2017 Sep 08 & 58004.38974 & FTS & $r^{\prime}$ & 300 \\
2017 Sep 08 & 58004.98666 & $1 \mathrm{~m}-\mathrm{C}$ & $y$ & 200 \\
2017 Sep 09 & 58005.44592 & FTS & $i^{\prime}$ & 200 \\
2017 Sep 09 & 58005.76490 & $1 \mathrm{~m}-\mathrm{S}$ & $y$ & 200 \\
2017 Sep 10 & 58006.43538 & FTS & $i^{\prime}$ & 200 \\
2017 Sep 10 & 58006.77602 & $1 \mathrm{~m}-\mathrm{S}$ & $g^{\prime} i^{\prime} y$ & $300,200,200$ \\
2017 Sep 11 & 58007.42086 & FTS & $r^{\prime} i^{\prime}$ & $2 \times 300,200$ \\
2017 Sep 12 & 58008.42079 & FTS & $i^{\prime}$ & 200 \\
2017 Sep 13 & 58009.36657 & FTS & $i^{\prime}$ & 200 \\
2017 Sep 13 & 58009.43914 & $1 \mathrm{~m}-\mathrm{A}$ & $y^{\prime}$ & 200 \\
2017 Sep 14 & 58010.79202 & $1 \mathrm{~m}-\mathrm{S}$ & $y^{\prime}$ & 200 \\
2017 Sep 21 & 58017.40310 & $1 \mathrm{~m}-\mathrm{A}$ & $i^{\prime} y$ & 300,300 \\
2017 Sep 21 & 58017.43811 & FTS & $i^{\prime} y$ & 300,300 \\
2017 Sep 22 & 58018.40711 & $1 \mathrm{~m}-\mathrm{A}$ & $i^{\prime} y$ & 300,300 \\
2017 Sep 23 & 58019.01422 & $1 \mathrm{~m}-\mathrm{C}$ & $i^{\prime} y$ & 300,300 \\
2017 Sep 25 & 58021.42373 & FTS & $i^{\prime} y$ & 300,300 \\
2017 Sep 25 & 58021.98683 & $1 \mathrm{~m}-\mathrm{C}$ & $i^{\prime} y$ & 300,300 \\
2017 Oct 02 & 58028.99327 & $1 \mathrm{~m}-\mathrm{C}$ & $i^{\prime} y$ & 200,200 \\
2017 Oct 03 & 58029.37504 & $1 \mathrm{~m}-\mathrm{A}$ & $i^{\prime} y$ & 200,200 \\
2017 Oct 04 & 58030.98582 & $1 \mathrm{~m}-\mathrm{C}$ & $i^{\prime} y$ & 200,200 \\
2017 Oct 06 & 58032.37651 & $1 \mathrm{~m}-\mathrm{A}$ & $i^{\prime} y$ & 200,200 \\
2017 Oct 07 & 58033.41709 & FTS & $i^{\prime} y$ & 300,300 \\
2017 Oct 13 & 58039.39960 & FTS & $i^{\prime} y$ & 300,300 \\
\hline
\end{tabular}

Note. FTS is the $2 \mathrm{~m}$ Faulkes Telescope South in Australia, 1m-A is a $1-\mathrm{m}$ Node in Australia, $1 \mathrm{~m}-\mathrm{C}$ is a $1 \mathrm{~m}$ node in Chile, and $1 \mathrm{~m} \mathrm{~S}$ is a $1 \mathrm{~m}$ node in South Africa.

high foreground extinction and led us to schedule only $i^{\prime}$ - and $y$-band observations from September 12. A detailed observation $\log$ can be found in Table 1.

The Faulkes/LCO data were reduced (debiased and flatfielded) using the LCO automatic pipeline. Photometry was performed using PHOT in IRAF. ${ }^{33}$ For photometric calibration of the $r^{\prime}$ and $i^{\prime}$ bands, we used four close-by stars listed in the VST Photometric $\mathrm{H} \alpha$ Survey of the Southern Galactic Plane and Bulge (VPHAS+) Data Release 2 (Drew et al. 2014, 2016). The $r, i$ Vega catalog magnitudes of these stars were converted to $r^{\prime}, i^{\prime} \mathrm{AB}$ magnitudes, which is standard for SDSS filters. We found no catalogs with $y$-band magnitudes of any field stars (Pan-STARRS does not cover this field). However, we performed log-log polynomial fits to the $g^{\prime}, r^{\prime}, i^{\prime}$, $J, H$, and $K$ spectral energy distributions (SEDs) of these four field stars using the VPHAS + and 2MASS catalogs, and we interpolated the fluxes of each star to the $y$-band frequency in order to estimate the $y$-band magnitudes of the four stars. The polynomial curves provided good approximations to the reddened, blackbody SEDs of the stars (Figure 1). In all filters, the errors are dominated by the signal-to-noise ratio $(\mathrm{S} / \mathrm{N})$ of

\footnotetext{
33 IRAF is distributed by the National Optical Astronomy Observatory, which is operated by the Association of Universities for Research in Astronomy, Inc. under cooperative agreement with the National Science Foundation.
} 


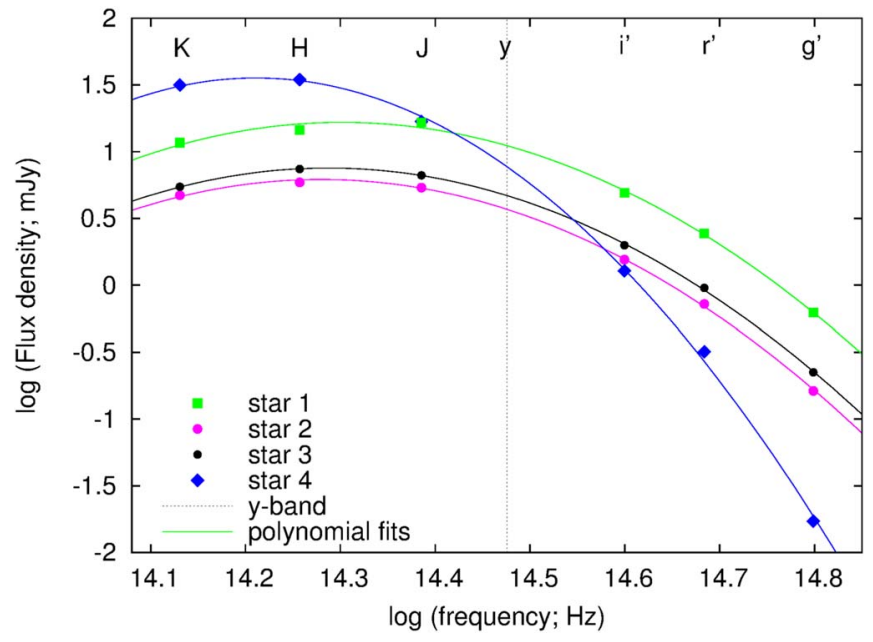

Figure 1. Polynomial fits to the optical-infrared SEDs of four field stars, used to calibrate the $y$-band LCO data. The vertical dotted line corresponds to the central frequency of the $y$-band filter.

the target (not the systematic calibration uncertainty). The LCO light curves are shown in the upper panel of Figure 2.

\subsubsection{REM Observations}

J1535 was observed with REM using the $g^{\prime} r^{\prime} i^{\prime} z^{\prime}$ SDSS filters of the ROSS2 instrument (4000-9500 $)$ ) between 2017 September 7 (MJD 58003) and October 1 (MJD 58027), obtaining strictly simultaneous images of the field. At the same time, thanks to the REMIR instrument, observations in the NIR ( $J, H$, and $K$ bands, 1.2-2.2 $\mu \mathrm{m}$ ) were also acquired. For each night of observations, the single shots were averaged in each band in order to enhance the $\mathrm{S} / \mathrm{N}$. In this period of time, a total of 17 NIR-optical quasi-simultaneous data sets were obtained with REM. A detailed log of the observations can be found in Table 2. Each optical image was bias-corrected and flat-fieldcorrected using standard procedures, and the magnitudes of all objects in the field were extracted using the point-spread function (PSF) photometry technique with the dedicated ESOMIDAS $^{34}$ daophot ${ }^{35}$ task. The target was detected in the $z^{\prime}$ and $i^{\prime}$ bands only, in three and one epoch, respectively, probably due to the high extinction of the field $\left(E_{\mathrm{B}-\mathrm{V}}=3.82\right.$; see Section 4.2 for more details). The flux calibration was performed against the standard star SA 110 (Smith et al. 2005).

NIR images were sky-subtracted, and the fluxes were extracted following the same procedure as in the optical. The NIR flux calibration was performed against a group of 12 isolated field stars in the $2 \mathrm{MASS}^{36}$ catalog (Skrutskie et al. 2006). The REM light curves are shown in the upper panel of Figure 2.

\subsection{MIR Photometry with VLT + VISIR}

MIR observations of the field of J1535 were made with the Very Large Telescope (VLT) on seven nights from 2017 September 12 to 23, under the program 099.D-0884 (PI: D. Russell). The VLT Imager and Spectrometer for the midInfrared (VISIR; Lagage et al. 2004) instrument on the VLT's UT3 (Melipal) was used in small-field imaging mode (the pixel

\footnotetext{
34 http://www.eso.org/projects/esomidas/

35 http://www.star.bris.ac.uk/ mbt/daophot/

36 http://www.ipac.caltech.edu/2mass/
}

Table 2

Detailed Log of the REM Optical (ROSS2) and NIR (REMIR) Observations Performed between 2017 September 7 and October 1

\begin{tabular}{lccccc}
\hline \hline \multirow{2}{*}{$\begin{array}{l}\text { Epoch } \\
\text { (UT) }\end{array}$} & \multicolumn{2}{c}{ MJD Mid Observation } & & \multicolumn{2}{c}{ Exposures (s) } \\
\cline { 2 - 3 } & ROSS2 & REMIR & & $g^{\prime} r^{\prime} i^{\prime} z^{\prime}$ & $J H K$ \\
\hline 2017 Sep 07 & 58003.08438 & 58003.08538 & & $3 \times 180$ & $5 \times 30$ \\
2017 Sep 08 & 58004.09137 & 58004.09236 & & $3 \times 180$ & $5 \times 30$ \\
2017 Sep 11 & 58007.06196 & 58007.06293 & & $3 \times 180$ & $5 \times 30$ \\
2017 Sep 12 & 58008.09050 & 58008.09149 & & $3 \times 180$ & $5 \times 30$ \\
2017 Sep 14 & 58010.08851 & 58010.08949 & & $3 \times 180$ & $5 \times 30$ \\
2017 Sep 15 & 58011.09541 & 58011.09640 & & $3 \times 180$ & $5 \times 30$ \\
2017 Sep 16 & 58012.10244 & 58012.10341 & & $3 \times 180$ & $5 \times 30$ \\
2017 Sep 21 & 58017.02715 & 58017.02814 & & $3 \times 180$ & $5 \times 30$ \\
2017 Sep 22 & 58018.04153 & 58018.04252 & & $3 \times 180$ & $5 \times 30$ \\
2017 Sep 23 & 58019.04846 & 58019.04944 & & $3 \times 180$ & $5 \times 30$ \\
2017 Sep 25 & 58021.04214 & 58021.04312 & & $3 \times 180$ & $5 \times 30$ \\
2017 Sep 26 & 58022.04907 & 58022.05006 & & $3 \times 180$ & $5 \times 30$ \\
2017 Sep 27 & 58023.05597 & 58023.05695 & & $3 \times 180$ & $5 \times 30$ \\
2017 Sep 28 & 58024.07427 & 58024.07526 & & $3 \times 180$ & $5 \times 30$ \\
2017 Sep 29 & 58025.08112 & 58025.08211 & & $3 \times 180$ & $5 \times 30$ \\
2017 Sep 30 & 58026.08806 & 58026.08906 & & $3 \times 180$ & $5 \times 30$ \\
2017 Oct 01 & 58027.09537 & 58027.09635 & & $3 \times 180$ & $5 \times 30$ \\
\hline
\end{tabular}

scale was 45 mas pixel $\left.{ }^{-1}\right)$. Three filters $\left(M, J 8.9, \mathrm{PAH}_{2}\right)$ were used on different dates spanning central wavelengths 4.85-12.13 $\mu \mathrm{m}$. The VISIR observing log, including photometric results, is reported in Table 3 . For each observation, the integration time on source was $1000 \mathrm{~s}$, composed of 22 nodding cycles. With chopping and nodding between source and sky, the total observing time was 1800-1900 s per observation.

The data were reduced using the VISIR pipeline in the gasgano environment. Raw images from the chop/nod cycle were recombined and sensitivities were estimated based on standard star observations taken on the same night in the same filters. Aperture photometry was performed using an aperture large enough to ensure small seeing variations did not affect the fraction of flux in the aperture. The standard stars used are listed in the final column of Table 3. The flux densities of $\mathrm{J} 1535$ in the table were measured from the resulting combined frames.

We found that J1535 was bright enough on the first four epochs to measure the flux density of the source in individual nodding cycles. We produced short-timescale light curves on these four dates; these are presented in Figure 3. Clear MIR variations are observed on the time resolution of the observations, which is 80-90 s. In order to test whether these variations are intrinsic to the source, we investigate if sky variations, changing conditions, or low $\mathrm{S} / \mathrm{N}$ could account for the apparent variability. The overall long-term and night-tonight stability of the photometric calibration of VISIR is described in Dobrzycka et al. (2012), and the short-term stability was investigated during commissioning. From these tests, under photometric conditions, the sky transparency variations were less than a few percent, and under clear conditions larger variability (up to $\sim 10 \%$ ) was measured, although it is unlikely that the timescale of MIR variability would be as short as minutes, as appears to be the case for $\mathrm{J} 1535$. Moreover, the flux of J1535 varies by a factor of $\sim 2-3$ on timescales of minutes, which is in clear excess of ( $\geq 20-30$ times greater than) the variations expected from different conditions. VISIR has been used to study night-to-night 
Table 3

Observation Log and Results of the Mid-infrared Photometry Performed on J1535 with the VISIR Instrument between 2017 September 12 and 23

\begin{tabular}{lccccc}
\hline \hline $\begin{array}{l}\text { Epoch (UT) } \\
\text { start time (MJD) }\end{array}$ & Filter & $\begin{array}{c}\text { Wavelength } \\
(\mu \mathrm{m})\end{array}$ & $\begin{array}{c}\text { Weather } \\
\text { conditions }\end{array}$ & Air mass & $\begin{array}{c}\text { Flux density } \\
\text { of J1535 (mJy) }\end{array}$ \\
\hline 2017 Sep 12 01:47:37 (58008.07474) & $J 8.9$ & 8.72 & Clear & $2.04-2.35$ & $25 \pm 2$ \\
2017 Sep 14 23:19:22 (58010.97178) & $M$ & 4.85 & Photometric & $1.37-1.46$ & $62 \pm 5$ \\
2017 Sep 15 23:29:54 (58011.97910) & PAH2_2 & 12.13 & Photometric & $1.41-1.51$ & $90 \pm 2$ \\
2017 Sep 17 00:53:36 (58013.03690) & $J 8.9$ & 8.72 & Photometric & $1.78-2.01$ & $55 \pm 4$ \\
2017 Sep 17 23:58:20 (58013.99884) & $M$ & 4.85 & Clear & $1.53-1.67$ & $30 \pm 3$ \\
2017 Sep 22 23:41:34 (58018.98721) & $J 8.9$ & 8.72 & Clear & $1.54-1.68$ & $1,4,5,6$ \\
2017 Sep 23 23:42:32 (58019.98787) & PAH2_2 & 12.13 & Clear & $1.56-1.70$ & 5,7 \\
\hline
\end{tabular}

Note. The reported fluxes are not dereddened. Standard stars are as follows: $1=$ HD198048, $2=$ HD161096, $3=$ HD163376, $4=$ HD000787, $5=$ HD156277, $6=\mathrm{HD} 211416,7=\mathrm{HD} 178345,8=\mathrm{HD} 133550$.

Table 4

Optical and Mid-IR Photometric Variability Properties of J1535 with SALT and VLT

\begin{tabular}{lcccccrrr}
\hline \hline $\begin{array}{l}\text { Epoch UT } \\
\text { (Sept. 2017) }\end{array}$ & MJD & Filter & $\begin{array}{c}\text { Time } \\
\text { resolution (s) }\end{array}$ & $\begin{array}{c}\text { Flux density } \\
\text { mean value (mJy) }\end{array}$ & $\begin{array}{c}\text { Standard } \\
\text { deviation (mJy) }\end{array}$ & $\begin{array}{c}\text { Stdev/mean } \\
(\%)\end{array}$ & $\begin{array}{c}\text { Fractional } \\
\text { rms }(\%)\end{array}$ & $\begin{array}{c}\text { Max/min } \\
\text { range factor }\end{array}$ \\
\hline 8 & 58004 & White & 30.4 & $\ldots$ & $\ldots$ & 4.7 & $3.8 \pm 2.7$ & 1.26 \\
14 & 58010 & $M$ & 83.8 & 62.4 & 11.9 & 19.1 & $17.2 \pm 8.4$ \\
15 & 58011 & PAH2_2 & 81.2 & 90.2 & 13.5 & 15.0 & $14.9 \pm 4.8$ \\
17 & 58013 & $J 8.9$ & 87.1 & 54.8 & 2.09 & 2.03 & 22.4 & $2.0 \pm 8.7$ \\
\hline
\end{tabular}

Note. We include mid-IR data on the three dates in which the source was significantly detected in all 22 nodding cycles.

variability in some objects (e.g., van Boekel et al. 2010), but we are not aware of any other published fast photometric variability studies using VISIR.

To further investigate if changing sky conditions could account for the apparent variability in J1535 at the time of the observations, we inspected the variations of the standard stars over the same dates. We found that the ADU/flux conversion factor measured from each standard star observation (in order to flux calibrate the data) varied by $7 \%-8 \%$ over different dates (from September 11 to 23) under different weather conditions (clear or photometric) at different air masses. The variations in conversion factor on the level of $7 \%-8 \%$ in the standards from night to night are likely due to differences in the sky conditions and air mass, as well as intrinsic differences between the conversion factors derived for different standard stars. J1535 clearly has a lower $\mathrm{S} / \mathrm{N}$ than the standard star observations, but the lower $\mathrm{S} / \mathrm{N}$ cannot account for the observed variations in J1535 since the error bars on each flux measurement (which take into account variations in the background) are much smaller than the difference between fluxes in the same 30-minute light curves (Figure 3). Possible background variations due to the water vapor content of the atmosphere above Paranal have moreover been monitored during our observations, revealing no clear variability of the background, which remained essentially constant during the observations. We therefore conclude that the MIR variations on minute timescales are almost certainly intrinsic to the source. In Table 4 we quantify the properties of the MIR variability of $\mathrm{J} 1535$ on the three dates in which the source was significantly detected in all 22 frames (these three dates were all under photometric conditions). We measured the intrinsic fractional rms variability (which subtracts the contribution to the variations from Poisson noise) by adopting the method of Vaughan et al. (2003) and Gandhi et al. (2010).

\subsection{Fast Optical Photometry with SALT}

J1535 was observed using the imaging camera SALTICAM (O'Donoghue et al. 2006) on the Southern African Large Telescope (SALT; Buckley et al. 2006) near the beginning of the outburst, on 2017 September 8 at 17:37-18:34 UT (MJD 58004.7). In the clear (white light) filter, 110 consecutive images of the field were made, each of exposure $30 \mathrm{~s}$. These observations were done in frame transfer mode, with no dead time between exposures. Automatic image reduction, star identification, and relative magnitude measurements were run under the PySALT pipeline (Crawford et al. 2010). J1535 was clearly detected in all frames. In Figure 4 we present the light curve, which shows the intrinsic variability of J1535. The light curve of a nearby, slightly fainter field star is also shown and is clearly much less variable. The variability properties are included in Table 4, adopting the same method as above to calculate the fractional rms variability. From differential measurements with USNO-B1.0 cataloged field stars, we estimated the brightness of MAXI at the time of the SALT observation to be $\sim 19$.

\section{Results}

\subsection{Optical and Infrared Light Curves: Variability}

The optical and NIR light curves obtained with the REM and LCO telescopes are shown in Figure 2 (upper panel), in comparison with the MIR light curves obtained with VLTVISIR (Figure 2, middle panel) and the Swift BAT 15-50 keV and MAXI 2-10 keV (Figure 2, bottom panel) light curves during the same period of time.

The transition from a hard to a softer state reported in Shidatsu et al. (2017b) on MJD 58015 is clearly visible in the Swift light curve as a significant decrease of the hard X-ray flux. Unfortunately, we do not possess an optical/NIR observation on that day, which falls in a gap of our data 

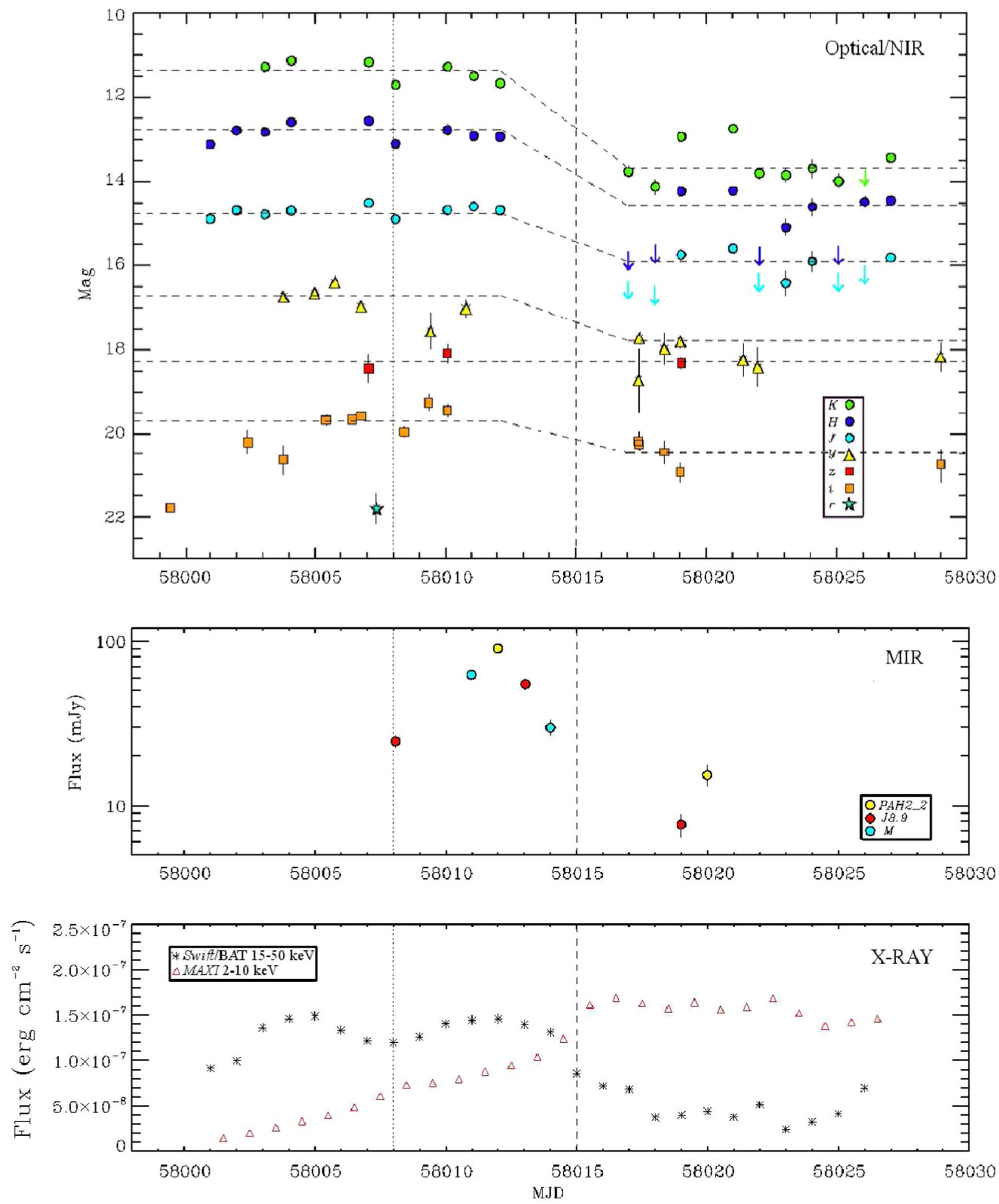

Figure 2. Upper panel: optical and NIR light curves of J1535 during its 2017 outburst, obtained with the REM (JHK and $i^{\prime} z^{\prime}$ bands) and Faulkes ( $r^{\prime} i^{\prime} y$ bands) telescopes from 2017 September 6 to October 3 (i.e., MJD 58002-58029). The SMARTS $J H$ detections reported by Dincer (2017) and the $i^{\prime}$-band detection reported by Scaringi (2017) are also shown (MJD 57999-58001). Errors are indicated at the 68\% confidence level. Magnitudes are not corrected for reddening. The 3 $\sigma$ upper limits are indicated using arrows, where needed. Superimposed are the fits of the light curves with constant functions before and after the drop, to highlight the change. The two flaring points in the $J H K$ band, the upper limits, and the first $i^{\prime}$-band point have been excluded from the fits. Middle panel: MIR light curves of J1535 obtained with the VISIR instrument, using the J8.9, $M$, and $P A H 2 \_2$ filters. The values of the flux densities are not dereddened. Bottom panel: light curve of J1535 obtained with the BAT instrument mounted on Swift (15-50 keV; black x), and with the MAXI instrument (2-10 keV; red triangles) from 2017 September 6 to October 13. Errors are indicated at the $68 \%$ confidence level. The drop in the hard X-rays, corresponding to an increase of the soft X-ray flux, is clearly visible at MJD 58015 (dashed line in all panels), and the brief, slight softening is seen before that at MJD 58008 (dotted line in all panels). 

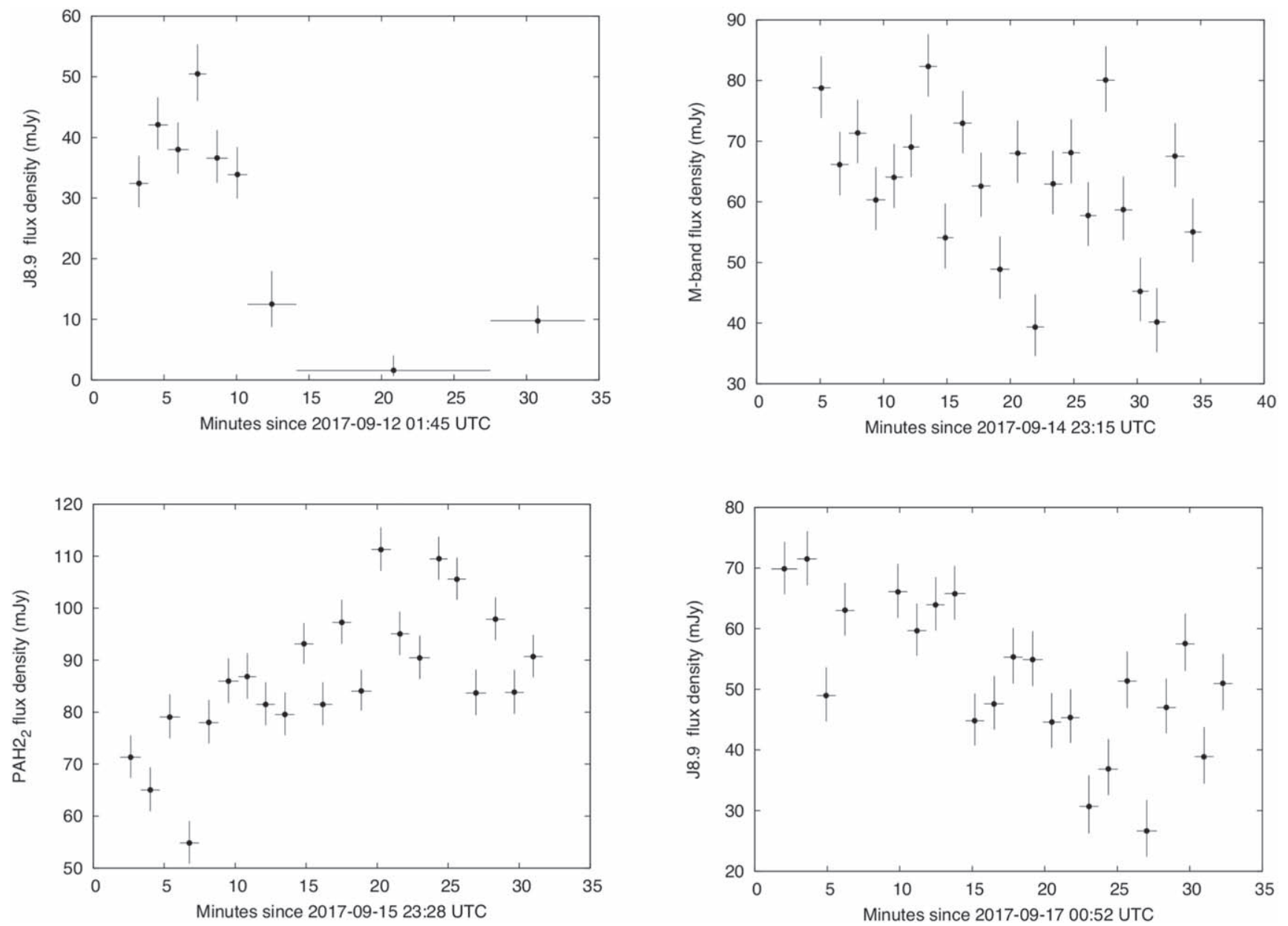

Figure 3. MIR light curves of J1535 obtained with VLT-VISIR at four epochs: September 12 (MJD 58008; J8.9 band), 14 (MJD 58010 ; $M$ band), 15 (MJD 58011 ; PAH2_2 band), and 17 (MJD 58013; J8.9 band).

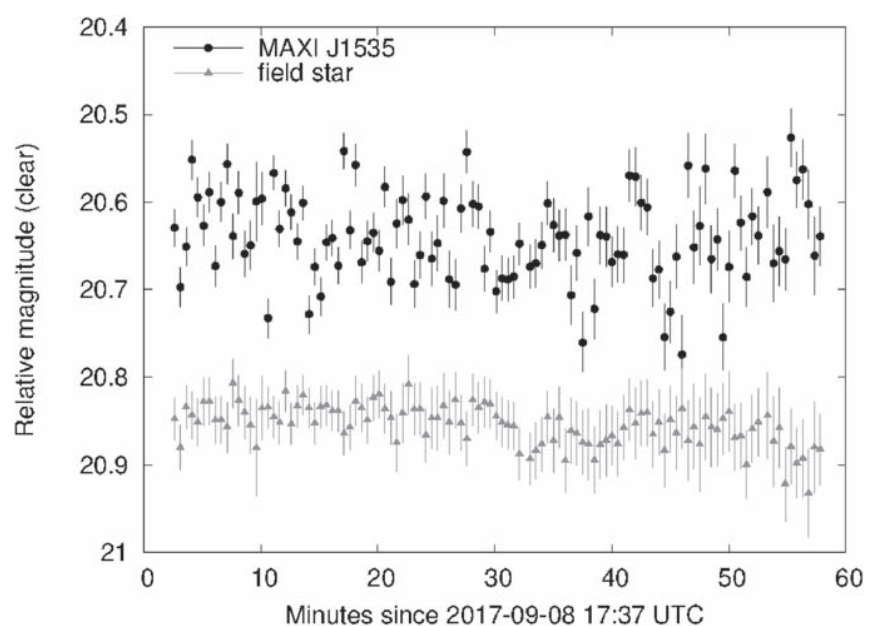

Figure 4. Optical (white light) light curve of J1535 obtained with SALTSALTICAM on September 8 (MJD 58004.734).

set; however, a large drop in the flux (more prominent going toward lower frequencies, reaching $\sim 2$ mag in the $K$ band; see Figure 5) is clearly noticeable between MJD 58012 and 58017, that is, around the same time of the X-ray spectrum softening. This frequency dependence of the fade suggests that, at least at NIR frequencies, the component that dominates the emission is likely to be the jet, which is quenched once the X-ray spectrum of the source softens, as expected in the case of BHBs (e.g., Homan et al. 2005; Coriat et al. 2009; Cadolle Bel et al. 2011; Russell et al. 2013b, 2014). Despite the flux drop, the light curves display large variability on daily timescales, with both intraobservation flares and dips during the whole monitoring. In the NIR in particular, variations up to $1.2 \mathrm{mag}$ in the $K$ band between two near epochs (i.e., on a timescale of $\sim 1$ day) are observed. These features are uncommon for $\mathrm{BH}$ candidate LMXBs, which normally show a single NIR drop when the source transitions to the soft state when the jet is quenched, and then again a single NIR rise at the end of the outburst, when the source goes back to the hard state (see, e.g., the case of GX 339-4, Homan et al. 2005; Coriat et al. 2009; Cadolle Bel et al. 2011; Buxton et al. 2012).

Also in the MIR, a dramatic fading of the flux density corresponding to the softening of the X-ray spectrum around MJD 58015 is observed. In particular, a flux decrease of a factor of 7 in the $J 8.9(8.72 \mu \mathrm{m})$ band between MJD 58008 and 58018 and of a factor of 6 in the PAH2_2 $(12.13 \mu \mathrm{m})$ band between MJD 58011 and 58019 is detected (see Figure 2, middle panel).

Moreover, a large amount of intrinsic variability is observed in the MIR (Figure 3). The source being very bright in several epochs (see Table 3), the $\mathrm{S} / \mathrm{N}$ in the observations was high enough to search for short-term (minutes) variations in the light 


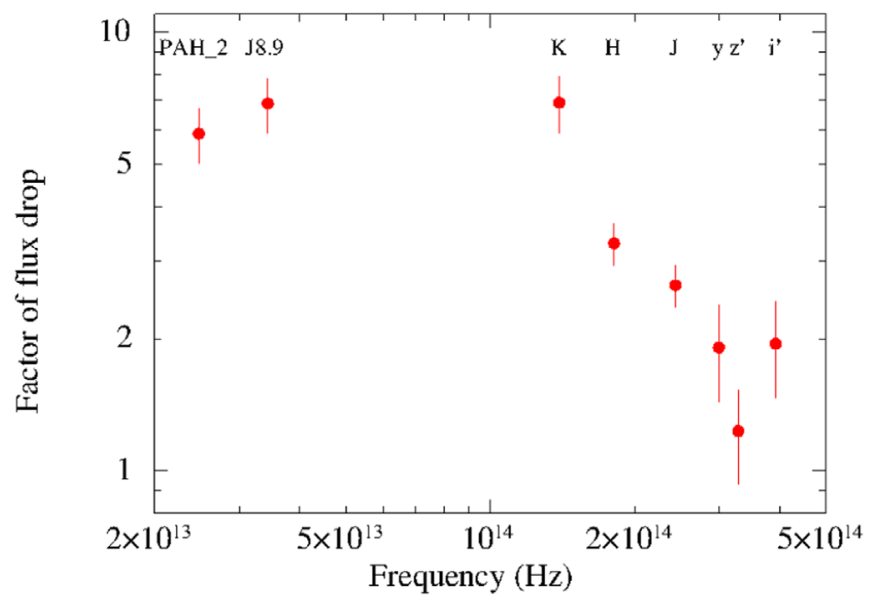

Figure 5. Flux drop from MIR to optical as a function of the frequency. The drop is defined as the ratio between the flux soon before and soon after the transition to the soft state.

curves obtained with the VISIR instrument. The brightest observation in particular (MJD 58011 in the PAH2_2 band) can be split into 22 images of $\sim 80 \mathrm{~s}$ time resolution each, and the flux appears to vary significantly, by a factor of $\sim 2$ in less than 15 minutes (see Figure 3). The measured fractional $\mathrm{rms}$ is $\sim 15 \%-22 \%$ (see Table 4), which is comparable to the fractional rms of GX 339-4 in the optical at a similar time resolution (Gandhi 2009; Gandhi et al. 2010) and in the $K$ band (13.20 $\pm 0.05 \%$; Vincentelli et al. 2018).

To investigate this variability further, we also searched for short-timescale variability in the NIR data set, by splitting the observations at higher $\mathrm{S} / \mathrm{N}$ into five different images of $30 \mathrm{~s}$ integration each. The NIR flux densities varied significantly on some of the dates, with a maximum variation of a factor of $\sim 2.5$ in less than 1 minute in the $H$ band on MJD 58008 . Interestingly, MJD 58008 corresponds to the epoch before the softening of the X-ray spectrum, in which the lowest flux was reported in our NIR campaign (see Figure 2), and shows light curves that are variable at a $3 \sigma$ confidence level. These NIR observations are simultaneous with the last part of our MIR J8.9 band observations, which showed a sudden decrease of the flux density to undetectable levels after the first $\sim 10$ minutes of a high detected flux ( $\sim 40 \mathrm{mJy})$. Interestingly, moreover, this temporary fading of the infrared emission corresponds to a dip in the hard X-ray light curve, that is, to a short-lived softening of the X-ray spectrum.

\subsection{Dereddening}

To obtain an estimate of the absorption column density and associated uncertainties, which is essential in order to deredden our infrared and optical fluxes and to build broadband spectra of the target, we first started by fitting the Swift/XRT spectrum of J1535 acquired during a $1.1 \mathrm{ks}$ observation carried out on 2017 September 11 (obs ID: 00010264004) with an absorbed power-law model. Pile-up effects were mitigated by excising the inner portion of the source PSF (within a radius of 12 arcsec). Absorption by the interstellar medium along the line of sight toward the source was modeled via the TBABS model (Wilms et al. 2000) with abundances from Anders \& Grevesse (1989). The fit was statistically acceptable, yielding a reduced $\chi_{\nu}^{2}=1.08$ for 715 degrees of freedom. The inferred value for the column density and power-law photon index were $N_{\mathrm{H}}=(2.62 \pm 0.02) \times 10^{22} \mathrm{~cm}^{-2}$ and $\Gamma=1.96 \pm 0.01$. This $N_{\mathrm{H}}$ leads to a $V$-band absorption coefficient $A_{V}$ of $9.13 \pm 0.50$ (Foight et al. 2016).

If we consider the abundances from Wilms et al. (2000) instead of those of Anders \& Grevesse (1989), we obtain a higher value of the $N_{\mathrm{H}}\left(3.84 \pm 0.03 \times 10^{22} \mathrm{~cm}^{-2}\right)$, which is consistent with what is reported in, for example, Kennea et al. (2017). With this $N_{\mathrm{H}}, A_{V}=13.38 \pm 0.73$ is inferred. The principal difference between the two abundances lies in the fact that Anders \& Grevesse (1989) used the solar abundances as the reference ones for the interstellar medium (ISM), while Wilms et al. (2000) used a more accurate estimate of the ISM abundances, even if still very uncertain (see Wilms et al. 2000 for further details). Anders \& Grevesse (1989) abundances typically tend to favor a lower value of $N_{\mathrm{H}}$, as we found.

The lowest value of the column density estimate for this source is instead given by the Galactic $N_{\mathrm{H}}$, which is $1.43 \times 10^{22} \mathrm{~cm}^{-2}$ in the direction of the source (Kalberla et al. 2005), from which we estimate $A_{V}$ of $4.98 \pm 0.27$ and $E_{B-V}$ of $1.61 \pm 0.09$. This suggests that the $N_{\mathrm{H}}$ measured from the modeling of the X-ray spectrum may have a strong component that is intrinsic to the source, and therefore may not be a good tracer of the dust absorption along the line of sight.

Moreover, Tao et al. (2018) reported on evidence of variable $N_{\mathrm{H}}$ during the days covered by our optical and infrared observations. However, if the local $N_{\mathrm{H}}$ is not due to the presence of dust, the $N_{\mathrm{H}} / A_{V}$ relation of Foight et al. (2016) is not applicable, meaning that a changing $N_{\mathrm{H}}$ does not necessarily affect the $A_{V}$ absorption. To verify if this is the case for MAXI J1535, we first considered a hypothetical source with constant infrared and optical fluxes, and we considered a possible changing $A_{V}$ that is due to the changing $N_{\mathrm{H}}$ (as reported in Tao et al. 2018). We then dereddened the constant fluxes of the source using these values of $A_{V}$, and we built new light curves. This test resulted in an optical $i^{\prime}$-band light curve that varied by $2.5 \mathrm{mag}$ (a factor of $\sim 10$ in flux), which is a higher amplitude than observed. This test also predicts the $K$-band light curve to be approximately constant but slightly brighter after the transition, whereas the opposite is observed (Figure 2).

We then searched for possible correlations between the infrared-optical colors measured for MAXI J1535 and the varying $N_{\mathrm{H}}$ measured from the modeling of the Swift spectra during the outburst (Tao et al. 2018), but no correlation was found. Therefore, we conclude that while the intrinsic $N_{\mathrm{H}}$ is changing wildly (as measured from X-ray spectra), there is no corresponding expected change in the infrared-optical flux or colors, so intrinsic $A_{V}$ is negligible.

Further arguments that could explain a lack of dust intrinsic to the source might be that (1) the absorber resides between the optical-IR and X-ray emission regions, or (2) the absorber is not large enough to cover the entire optical-IR emitting region, or (3) the absorber may be dust-free or have a very low dust-togas ratio. Oates et al. (2018) recently discovered that in V404 Cyg (which had such a high variable $N_{\mathrm{H}}$ that it sometimes was Compton thick), the dust-to-gas ratio must be on the order of $10^{4}$ smaller than that typically observed in the Milky Way, in order to not show significant change in color as the absorption increased by a factor of 1000 . The absorber has to be within or at least close to the dust sublimation radius in order to be dustfree. Oates et al. (2018) found that for V404 Cyg, the X-ray 
luminosity of V404 in quiescence resulted in a dust sublimation radius comparable to the size of the accretion disk. V404 Cyg is a system with a long orbital period, so it is extremely likely that for MAXI J1535 in outburst, with orders-of-magnitude higher X-ray luminosity than V404 Cyg in quiescence, the dust sublimation radius will be orders of magnitude outside the orbital separation of the X-ray binary. On the other hand, the intrinsic $N_{\mathrm{H}}$ is likely to originate from an absorber located very close to the black hole (as was the case in V404 Cyg; see, e.g., Motta et al. 2017), since it is variable on short timescales.

\subsection{Broadband Spectral Evolution}

The difficulty in determining the dust absorption coefficients in the direction of the source introduces large uncertainties when constructing the dereddened optical-IR (OIR) SEDs for J1535. Due to the high variability of the source, moreover, building an average spectral energy distribution of the target on a particular date is challenging. We therefore searched for observations that were as simultaneous as possible, in order to overcome at least this last issue.

Thanks to the REM telescope, we possessed simultaneous data sets in the optical, where unfortunately the target is barely detected only in the reddest bands due to the high extinction of the field. The NIR REM images are almost simultaneous with our optical observations (see Table 2), and in three epochs (MJD $58008,-11,-12)$, we also have MIR observations that are very near in time to the REM ones (see Table 3; in particular, we notice that the last part of the MJD 58008 MIR observation overlaps with the NIR one). Therefore, we can construct the OIR SEDs at least on these three epochs, bearing in mind that the contribution of the jet might produce significant changes in the SED over timescales of minutes, as observed in the MIR light curves. The three SEDs on MJD 58008, 58011, and 58012 (2017 September 12,15 , and 16 , respectively), dereddened using three different values of $N_{\mathrm{H}}\left(1.43 \times 10^{22} \mathrm{~cm}^{-2} ;(2.62 \pm 0.02) \times\right.$ $10^{22} \mathrm{~cm}^{-2}$; $(3.84 \pm 0.03) \times 10^{22} \mathrm{~cm}^{-2}$; see Section 4.2), are shown in Figure 6.

As is clearly visible in Figure 6, considerable changes in the shape of the SEDs are obtained by modifying the value of $N_{\mathrm{H}}$ used to deredden the fluxes. Using the lowest estimate of $N_{\mathrm{H}}$, the NIR-optical spectrum is described by a power law with a very steep slope ( $\alpha \sim-3)$, which is difficult to interpret. In fact, if we link the OIR emission of the system to the presence of a jet, we would expect to observe in the NIR-optical an optically thin synchrotron spectrum, which is normally described by a less-steep power law than what we observe (typically $\alpha \sim-0.8$; see, e.g., Gandhi et al. 2011). Instead, if the accretion disk is contributing to the NIR-optical emission, we would expect to observe a positive slope, due to the blackbody tail of the disk, which is clearly not our case. This is therefore an indication that the galactic $N_{\mathrm{H}}$ is probably an underestimate of the real absorption of light on the target line of sight.

The second estimate of $N_{\mathrm{H}}\left(2.62 \pm 0.02 \times 10^{22} \mathrm{~cm}^{-2}\right.$; Figure 6, middle panel) gives instead SEDs that are not extremely steep (except for MJD 58008, where the $i$-band flux is really low with respect to the lower frequency points), with a slope of $\sim-1.5$ and -0.8 on MJD 58011 and 58012, respectively. However, the contribution of the accretion disk is still missing at the highest frequencies, which is uncommon for an X-ray binary in outburst.

Instead, using $N_{\mathrm{H}}=3.84 \pm 0.03 \times 10^{22} \mathrm{~cm}^{-2}$, the contribution of the disk starts to be evident, and a clear infrared excess due to the emission of a jet is visible (see Figure 6, right panels). In particular, the slope of the power law that we used to fit the lower frequency points of the MJD 58011-12 SEDs are $\sim-0.8$ to -0.6 , respectively, which are values that are typically measured for the optically thin synchrotron spectrum of a jet.

For this reason, from now on, the value of $N_{\mathrm{H}}$ of $3.84 \pm 0.03 \times 10^{22} \mathrm{~cm}^{-2}$ will be used to deredden the fluxes of $\mathrm{J} 1535$. The corresponding absorption coefficients evaluated for all wavelengths that are relevant in this work are reported in Table 5. However, we caution the reader that the dereddened spectral slopes depend critically on the uncertain extinction.

Focusing on the right panel of Figure 6, we notice that on all dates the negative-index power law extends up to the MIR. If we interpret it as optically thin synchrotron emission from the jet, this suggests that the jet break frequency might fall at lower frequencies than the MIR, that is, in the radio to far-IR bands (with upper limits to the jet break frequency of $6.2 \times 10^{13} \mathrm{~Hz}$ and $2.5 \times 10^{13} \mathrm{~Hz}$ on MJD 58011 and 58012, respectively). On MJD 58008, the slope of the SED at lower frequencies is lower with respect to MJD 58011 and 58012, which suggests a lower contribution of the jet at those frequencies on that day. However, we caution that MJD 58008 is the epoch in which the MIR observation shows the sudden drop in flux after the first $\sim 15$ minutes of observation, until it becomes undetectable. This strong, short-timescale MIR variability might therefore distort the low-frequency shape of the SED at this epoch. Moreover, Tetarenko et al. (2017) reported on radio detections of J1535 with ALMA on the same day, with a preliminary flux measurement of $\sim 220 \mathrm{mJy}$ at 7 and $140 \mathrm{GHz}$, which is much higher than what we measure in our J8.9-band VISIR observation ( $\sim 63 \mathrm{mJy}$ after dereddening).

In Figure 7, all of the nearly simultaneous SEDs collected in our campaign are shown, from red to purple following the rainbow colors (as explained above, $N_{\mathrm{H}}=(3.84 \pm 0.03) \times$ $10^{22} \mathrm{~cm}^{-2}$ has been used to deredden the fluxes). The drop in the NIR flux that is observed in the light curves (see Figure 2) after MJD 58012 is clearly noticeable in the SEDs, with a large modification of the NIR SED shape between MJD 58012 and MJD 58019. In particular, if we fit the NIR-optical SEDs with a power law, the slope evolves from almost flat to positive (see Figure 7), with a residual infrared flux in the MIR, which might show that the contribution of the jet is lower but still detectable at the lowest frequencies, even if the X-ray spectrum softened (as reported in Shidatsu et al. 2017b). Also in the optical, the small drop in flux due to the decrease of the jet contribution is visible in the SEDs. A small drop is expected if the accretion disk has a stronger contribution to the optical than to the NIR.

If we then look at the MIR part of the SEDs, we can observe the fluxes changing considerably between the different epochs. The highest average flux is reached in the PAH2_2 band $(12.13 \mu \mathrm{m})$, on MJD 58012, with a dereddened value of $157 \pm 4 \mathrm{mJy}$; after that, the average MIR flux decreases, reaching its lowest value on MJD 58020 ( $27 \pm 4 \mathrm{mJy})$. The first measurement after the softening of the X-ray spectrum is a dereddened flux density in the $J 8.9$ band of $20 \pm 3 \mathrm{mJy}$ (MJD 58019), which is a factor of 7 lower than the flux density measured just before the softening in the same band (i.e., $141 \pm 12 \mathrm{mJy}$ on MJD 58013). It is therefore evident that we are observing in the MIR the emission of a transient component, which is suppressed as the X-ray spectrum softens, as usually happens in the case of jets quenching over the hardto-soft transition in BHBs. 

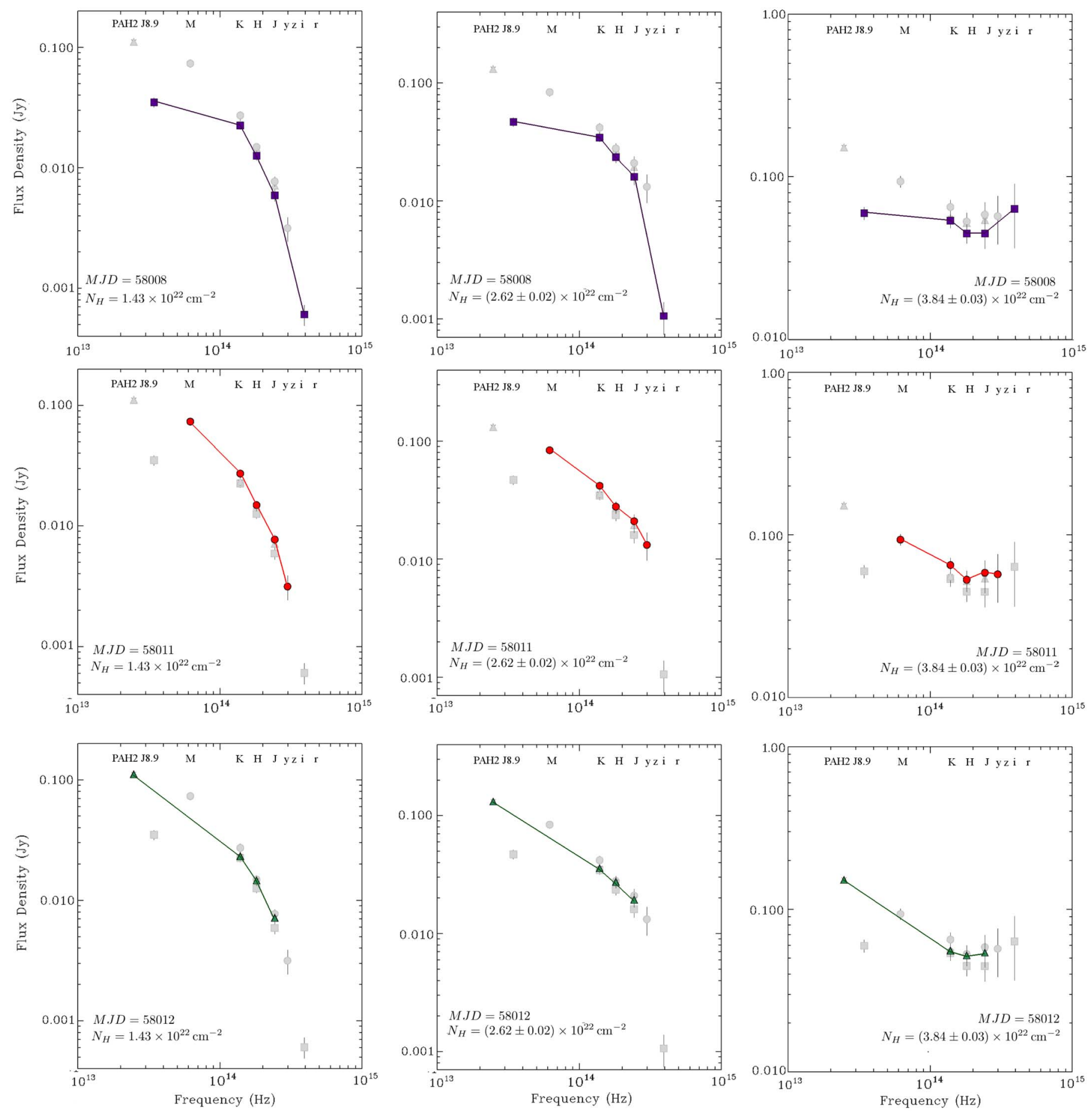

Figure 6. Nearly simultaneous OIR SEDs of J1535 on MJD 58008 (purple squares), 58011 (red dots), and 58012 (green triangles), obtained after dereddening using the galactic $N_{\mathrm{H}}\left(1.43 \times 10^{22} \mathrm{~cm}^{-2}\right.$; left panel), $N_{\mathrm{H}}=2.62 \pm 0.02 \times 10^{22} \mathrm{~cm}^{-2}$ (middle panel), and $N_{\mathrm{H}}=3.84 \pm 0.03 \times 10^{22} \mathrm{~cm}^{-2}$ (right panel). In each panel, all three SEDs are represented for comparison purposes. Errors are shown at the $68 \%$ confidence level.

\section{Hardness-Intensity Diagram}

We constructed the hardness-intensity diagram (HID) of J1535 (Figure 8) in order to study the evolution of the X-ray spectrum of the target during the first part of its outburst (from MJD 58001 to MJD 58139, i.e., 2017 September 5 to 2018 January 21), and to test whether the IR flaring episodes correspond to changes in the evolution in the HID. We considered the MAXI 2-10 keV count rate obtained almost daily during the monitoring of the outburst as an estimate of the soft X-ray photons, and the Swift/BAT (15-50 keV) count rate for the hard ones, and we evaluated the X-ray color (or hardness ratio, HR) as the ratio between the two. The count rates were converted into flux measurements using the WebPIMMS $^{37}$ tool.

During the rise of the outburst, until MJD 58015, the source shows a hard X-ray spectrum, and, as already pointed out in this

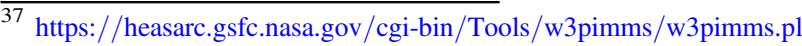




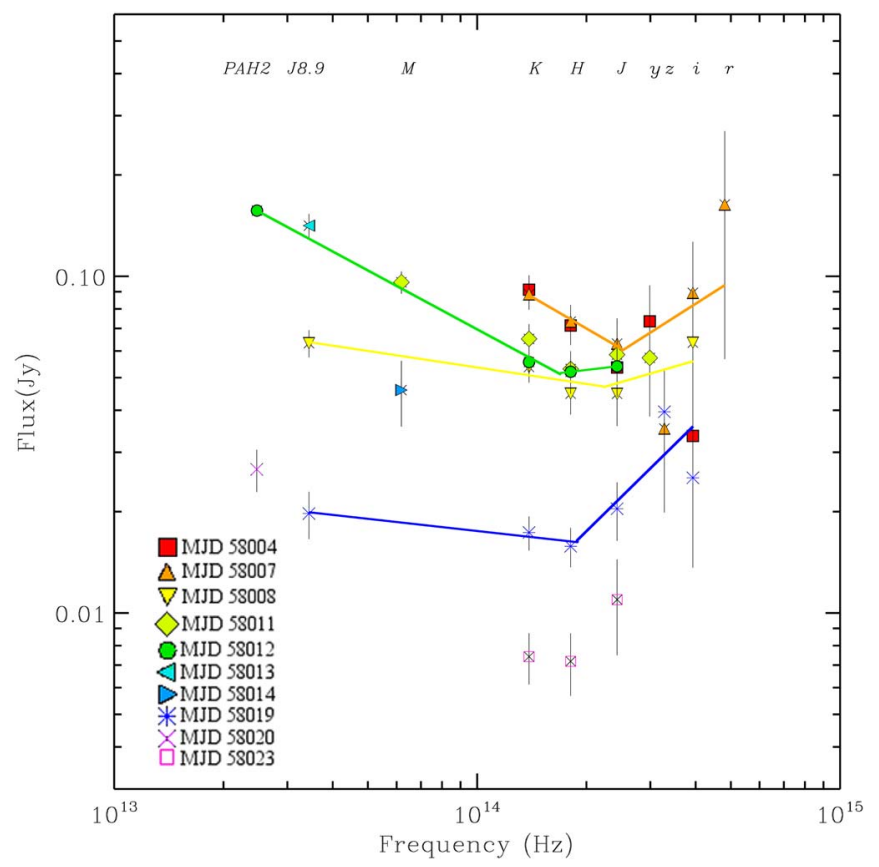

Figure 7. Spectral energy distributions of J1535 from MJD 58004 to MJD 58023. The SEDs evolve from red (MJD 58004) to purple (MJD 58023). The flux densities at all wavelengths have been dereddened using the absorption coefficients reported in Table 5. Errors are reported at the $68 \%$ confidence level.

Table 5

Absorption Coefficients

\begin{tabular}{lcc}
\hline \hline Filter & $\begin{array}{c}\text { Wavelength } \\
(\mu \mathrm{m})\end{array}$ & $\begin{array}{c}A_{\lambda} \\
(\mathrm{mag})\end{array}$ \\
\hline PAH2_2 & 12.13 & $0.60 \pm 0.02$ \\
$J 8.9$ & 8.72 & $1.03 \pm 0.03$ \\
$M$ & 4.85 & $0.47 \pm 0.02$ \\
$K$ & 2.16 & $1.51 \pm 0.08$ \\
$H$ & 1.66 & $2.20 \pm 0.12$ \\
$J$ & 1.23 & $3.51 \pm 0.19$ \\
$y$ & 1.00 & $5.01 \pm 0.27$ \\
$z^{\prime}$ & 0.91 & $5.91 \pm 0.32$ \\
$i^{\prime}$ & 0.76 & $8.05 \pm 0.44$ \\
$r^{\prime}$ & 0.62 & $10.96 \pm 0.60$ \\
\hline
\end{tabular}

Note. Absorption coefficients derived from $N_{\mathrm{H}}=3.84 \pm 0.03 \times 10^{22} \mathrm{~cm}^{-2}$ and using the relation between $N_{H}$ and $A_{V}$ reported in Foight et al. (2016), and the coefficients reported in Schlafly \& Finkbeiner (2011) and Weingartner \& Draine (2001) for the NIR-optical and the mid-infrared wavelengths, respectively.

discussion, very bright radio detections of flat or inverted spectrum jets have been reported (Russell et al. 2017b; Tetarenko et al. 2017). These are typical features of BHBs in outburst and are all accounted for in the Fender et al. (2004) model for the accretion-ejection coupling mechanism. Following the X-ray state classification reported in Tao et al. (2018) in particular, MAXI J1535 underwent a first state change from the hard state (red dots in Figure 8) to a hard-intermediate state (HIM; green dots in Figure 8) between MJD 58004 and 58007. This smooth change of the spectrum hardness is also visible in Figure 8. At MJD 58015, the spectrum undergoes a first softening (as reported in Shidatsu et al. 2017b). The same softening of the X-ray spectrum is also reported in Tao et al. (2018) using Swift observations. According to them, on MJD 58015 the source entered the so-called soft-intermediate state (SIM; violet dots in Figure 8), where it remained until MJD 58050, that is, for the whole duration of our infrared-optical campaign. According to the accretionejection coupling scenario, this intermediate phase should correspond to the NIR fading, as observed in other BHB candidates when they start to soften (see, e.g., GX 339-4; Homan et al. 2005; Coriat et al. 2009; Cadolle Bel et al. 2011). This is exactly what we observe for $\mathrm{J} 1535$.

Then, just four days after the softening, on MJD 58019 we detect the already mentioned flickering activity in the NIR light curves, with a brightening by more than 1 mag (i.e., an increase of the flux by a factor of 2.5) on a one-day timescale in the $K$ band (and the source became visible again in the $J$ and $H$ bands after the drop). The enhanced activity in the NIR lasted at least two days, until MJD 58021. In the HID shown in Figure 8, we notice that this few-day period corresponds to a change in the hardness of the system, with a temporary inversion of the decreasing trend of the hardness (see the inset in Figure 8). After MJD 58022, the hardness started again to decrease. Similar excursions of the hardness are also observed later, up to about MJD 58051, that is, when the source finally entered its soft state. Unfortunately, we do not possess an NIR coverage with REM after October 1 (i.e., MJD 58027) to show a possible NIR counterpart to these hardness changes, since the source was no longer visible from La Silla. J1535 did not make a smooth transition to the soft state. The excursions in the HID starting around MJD 58018 appear to be associated with IR variations from an intermittent jet.

\section{Discussion}

In this paper we present an analysis of the optical-infrared (MIR and NIR) light curves and SEDs of J1535 during the first part of its 2017-2018 outburst.

\subsection{Jet Suppression after the X-Ray Spectrum Softening}

As shown in Figure 2, the NIR and optical fluxes stay at a high level during the first phases, which correspond to the rise of the X-ray outburst; at the same time, the X-ray spectrum was reported to be hard by several authors (see Section 2), as typically happens during the rise of a BHB X-ray outburst. After MJD 58015, however, that is, when Swift detected a first softening of the spectrum (Shidatsu et al. 2017b), the infrared and optical fluxes underwent a prominent drop, which indicates that one of the players in the infrared-optical emission was experiencing a suppression in correspondence to the softening of the X-ray spectrum.

From studies of the coupling between accretion and ejection in BHBs, it is clear that a compact jet is produced during the hard state of BHB outbursts, which is then quenched as the system passes to the soft state, due to the observation of a strong suppression of the radio emission after the transition (see Tananbaum et al. 1972; Fender et al. 1999, 2004; Corbel et al. 2004). Less clear is whether the same effect should also be visible at higher frequencies, where the contribution of the jet should not always dominate the emission. However, several cases in which this happens have been observed so far (see, e.g., Homan et al. 2005; Russell et al. 2006, 2013a; Coriat et al. 2009). In particular, if the jet largely contributes to the IR-optical emission, it is likely that we will observe a weakening of the IR-optical flux during a hard-to-soft state transition, which should be more prominent toward lower 


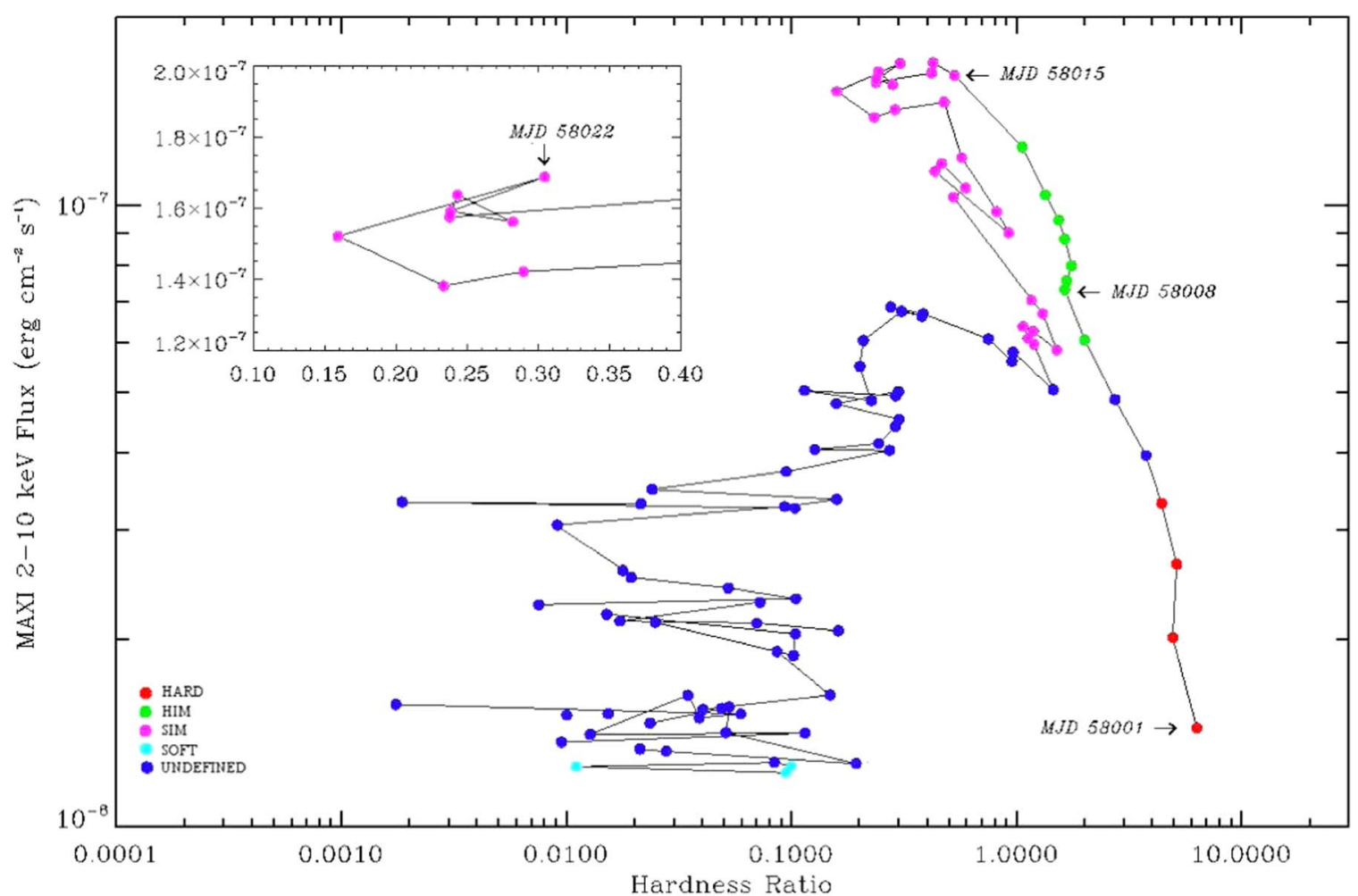

Figure 8. HID for the 2017-2018 outburst of J1535. The color code follows the state definition of Tao et al. (2018), according to which four principal states can be observed: the hard state (red dots), the hard-intermediate state (green dots), the soft-intermediate state (violet dots), and the soft state (light blue dots). In the figure, the first softening of the X-ray spectrum on MJD 58015, which corresponds to the drop in the OIR fluxes that we report in this work, is highlighted. The inset shows instead the sudden increase in the hardness on MJD 58022, which might correspond to the flares that we detect in the NIR light curves.

frequencies, where the contributions of the outer accretion disk and of the X-ray reprocessing are typically lower. This is exactly what we observe: the flux drop corresponding to the softening of the X-ray spectrum is in fact higher in the MIR and in the NIR, where we have a decrease of a factor of 6 at $12.13 \mu \mathrm{m}$ on an eight-day timescale, than in the optical, where the measured drop corresponds to $0.91 \mathrm{mag}$ in the $i^{\prime}$ band (i.e., a factor of $\sim 2$ in flux; see Figure 5).

If we follow the jet suppression interpretation to explain the flux drop that we observe, this implies that the jet was contributing at least $83 \% \pm 4 \%$ of the flux in the PAH2_2 band $(12.13 \mu \mathrm{m}), 86 \% \pm 9 \%$ in the $K$ band, and $57 \pm 21 \%$ in the $i^{\prime}$ band. This is consistent with what was found in Russell et al. (2006) for a sample of BHBs, for which the jets have been found to contribute $~ 90 \%$ of the NIR emission and below $76 \%$ of the optical emission in the hard state.

Inspecting the SEDs (Figure 7), we see that before the softening occurred on MJD 58015 in the IR regime, the spectrum is described by a power law with a negative index, with slopes ranging from -1 to -0.6 , which are typical of optically thin synchrotron emission from a jet (adopting $N_{H}=(3.84 \pm 0.03) \times$ $10^{22} \mathrm{~cm}^{-2}$ ). In the optical, the slope of the power law is always positive, but it becomes steeper after the drop, which shows that the accretion disk contribution becomes more important than the emission of the jet at those frequencies after the drop. This is also further evidence of the fact that the jet is contributing not only in the radio band, as reported by Russell et al. (2017b) and Tetarenko et al. (2017), but also at higher frequencies, up to the optical band, with the jet break frequency probably lying at lower frequencies than the MIR. We therefore interpret the observed drop in the IR-optical flux as the weakening of the emission of the jet while the X-ray spectrum softens.

\subsection{The Flickering Nature of the Jet in J1535}

The fade of the IR-optical flux near the start of the transition toward the soft state has already been observed in other BHBs. After the drop, the IR flux usually does not increase again, until the source transitions back to the hard state in the last phases of the outburst. Indeed, this was the case for XTE J1550-564 (Jain et al. 2001; Russell et al. 2010), MAXI J1836-194 (Russell et al. 2013a; Russell et al. 2014), and from repeated outbursts of GX 339-4 (Coriat et al. 2009; Buxton et al. 2012; Dinçer et al. 2012; see also Kalemci et al. 2013; Corbel et al. 2013). However, here we find that after the softening in $\mathrm{J} 1535$, the optically thin component seems to be still present in the MIR, even if strongly suppressed. In addition, inspecting the infrared light curves (Figure 2), we see the NIR flux experiences a sudden increase on MJD 58019-21, which does not have any correspondence with the optical light curves. This excludes that this flaring NIR activity might originate in the reprocessing of the accretion disk emission. It is therefore likely that the origin of these NIR flares is in the jet, which might experience a reflaring after the target started to soften. This hypothesis is supported by the SEDs, where it is clear that after the softening the jet seems to be still present at lower frequencies (MIR). Nearly simultaneous observations in the radio band might confirm or refute this interpretation.

As described in the semiquantitative model of Fender et al. (2004) and Fender et al. (2009; see also Vadawale et al. 2003), when a BHB is in a transitional phase between the hard and the soft state, it is likely to produce several synchrotron spectra from discrete ejections, which evolve toward lower frequencies with time, as the compact jet becomes more intermittent. This is seen most prominently at radio frequencies, later during the 
transition, close to the "jet line" when bright, optically thin radio flares are commonly observed. Multiple radio flares have been observed when BHBs perform hard-soft excursions in the HID during intermediate states. For example, GRS 1915+105 undergoes small loops in its HID that are accompanied by many radio flares from multiple ejections (Fender et al. 2004). Here, we instead detect IR flaring at earlier (harder) stages of the spectral transition.

In addition to the flaring after the main IR fading, we also notice that on MJD 58008 (hours before our infrared observations), ALMA millimeter and ATCA radio observations have been reported (see Tetarenko et al. 2017; Section 2), revealing that the flux in the millimeter band is found at a much higher value than what we measure in the MIR $(>200 \mathrm{mJy}$ at 97 and $140 \mathrm{GHz}$, versus $\sim 63 \mathrm{mJy}$ in the $J 8.9$ band of VISIR after dereddening). As described in Section 4.1, the MIR light curve that we extracted for the MJD 58008 epoch is highly variable, the source becoming undetectable after a certain time. Interestingly, the same decrease in the flux is also observed in the NIR on the same date, which on that day is strictly contemporaneous with the last part of the MIR observations (i.e., when the flux has already decreased). This behavior could again be interpreted as an intermittent jet, as well as flares within the jets, and appears to be associated with a small excursion in the HID, before the main softening.

\subsection{Short Time Variability: Possible Interpretation}

J1535 is the first BHB for which mid-infrared short time variability has been observed so far (with $\mathrm{rms} \sim 15 \%-22 \%$; Table 4). It is suggestive of the presence of a flickering jet, similar to what was found from MIR observations of GX 339-4 on longer (hour) timescales (Gandhi et al. 2011). Similar levels of fractional rms were observed in other BHBs at higher frequencies, like in the case of GX 339-4 in the optical (Gandhi et al. 2010). In that case, a study of the correlation between optical and X-ray (fast and slow) variability allowed the authors to exclude the accretion disk radiation reprocessing as the cause of the observed $\sim 15 \%$ optical rms, which was moreover higher for redder frequencies. Similar to what was suggested by Gandhi et al. (2010), a model that relates possible perturbations in the accretion flow to variability in the jet might explain the observed variability of our light curves. Such a model has been developed in Malzac (2013) and Malzac (2014; see also Drappeau et al. 2017). In this scenario, a strongly variable accretion flow could inject nonnegligible velocity fluctuations at the base of the jet, which would drive internal shocks at large distances from the $\mathrm{BH}$, where leptons are accelerated, giving rise to strongly variable synchrotron emission, which would in turn affect the radio-infrared emission of the system. This model has been found to be compatible with the MIR variability and SED observed by Gandhi et al. (2011) in GX 339-4 (Drappeau et al. 2017). We have found that the strong observed infrared variability is correlated with changes in the X-ray emission of the system on long timescales (days).

A detailed quantitative comparison of the consistency of the internal shocks model (Malzac 2013, 2014) with our observations goes beyond the scope of this paper. However, a comparison between what we observe and the expectations from the model can be easily performed. Using Figure 6 of Malzac (2014), we can infer the value of the fractional rms that is expected for optical and infrared light curves in a BHB with typical jet parameters (average Lorentz factor $\gamma=2$ for a kinetic power $P=1.3 \times 10^{37} \mathrm{erg} \mathrm{s}^{-1}$, jet half-opening angle $\Phi=1^{\circ}$, mass of the BH $M=10 M_{\odot}$, and so on; see Section 4 of Malzac 2014 for more details). In particular, it is clear from the figure that the expected optical rms should be higher than the midinfrared one in the Fourier frequency range that spans from the frequency corresponding to the total duration of each of our MIR light curves ( $~ 30$ minutes, out of scale in Figure 6 of Malzac 2014) to that associated with the time resolution ( $\sim 85 \mathrm{~s}$; see Table 4$)$. In the MIR, the fractional rms predicted by the model is $\sim 17 \%$ at a Fourier frequency of $1.2 \times 10^{-2} \mathrm{~Hz}$, which corresponds to the time resolution of our MIR observation for which the most significant rms has been detected $(81.2 \mathrm{~s}$ with the PAH2_2 band on September 15; see Table 4). Interestingly, this value is consistent with our findings (rms $\sim 15 \%$; Table 4$)$. In the optical, instead we obtain from the SALT light curve a much lower rms than expected (only 3\%-4\% rms; see Table 4). However, since J1535 is a BHB in outburst, it is likely that the accretion disk is playing a major role in the optical, thus diluting the jet emission at those frequencies, and therefore decreasing the detected fractional rms due to the internal shocks in the jet. A more detailed calculation of the expected rms should be performed by tailoring a simulation for $\mathrm{J} 1535$ so that it can reproduce the SED of the target. However, we do not expect the predicted rms to vary dramatically with the model parameters.

\section{Conclusions}

In this work, we present optical and infrared observations of the newly discovered $\mathrm{BH}$ candidate transient X-ray binary MAXI J1535-571 during the first phases of its recent outburst, which started on MJD 57998 (2017 September 2).

During the rise of the outburst, X-ray observations revealed a hard spectrum, as typical of BHBs, while the NIR and optical fluxes remained high. During this period of time, until MJD 58015 (September 19), the infrared spectral energy distribution was consistent with a power law with a negative index, likely due to the presence of a jet, as observed in the radio band. On MJD 58015, Swift detected a softening of the spectrum, which coincided with a drop of the infrared and optical fluxes by a factor of 10 and 2, respectively, on a timescale of a few days. The drop was more significant at lower frequencies, and we interpreted it as being due to the suppression of the emission of the jet, which is more dominant in the infrared than in the optical. A further confirmation of this scenario came from our mid-infrared campaign, performed with the VISIR instrument on the VLT. In fact, the mid-infrared fluxes also experienced a significant drop (by a factor of $\geqslant 6$ ) in correspondence to the observed softening of the X-ray spectrum, thus reinforcing our hypothesis.

The mid-infrared light curves have been found to possess a significant variability over a timescale of a few minutes, with a maximum measured $\mathrm{rms}$ of $\sim 15 \%-22 \%$. This makes MAXI J1535-571 the first BHB for which a mid-infrared shorttimescale variability study has been performed so far. This strong variability suggests the presence of a flickering jet, as previously observed in other BHBs using NIR-optical data (Casella et al. 2010; Gandhi et al. 2016, 2017; Kalamkar et al. 2016; Shahbaz et al. 2016; Vincentelli et al. 2018). The measured values of rms in the mid-infrared might be consistent with the internal shock model developed for jets in X-ray binaries (Malzac 2013, 2014). We also report a reflaring in the NIR light curves, after the main suppression of the jet. These 
late synchrotron flares are associated with X-ray hardness variations, which may be consistent with a jet spectral break frequency correlating with the X-ray hardness (Koljonen et al. 2015).

A comparison of our results with lower-frequency data (i.e., the radio band) is essential in order to clarify the proposed scenario and will be part of a follow-up paper on the outburst of this source.

The observations of rapid MIR flickering in J1535 open up a new field: studying the fast MIR variability in X-ray binaries. Such studies performed at optical and NIR wavelengths, especially conducted simultaneously with X-ray observations (e.g., Gandhi et al. 2010, 2017; Casella et al. 2010; Durant et al. 2011; Lasso-Cabrera \& Eikenberry 2013; Kalamkar et al. 2016), have led to leaps forward in our understanding of disk/jet physics. However, with current MIR instrumentation, it is not possible to observe BHBs (or NS LMXBs) at time resolutions less than minutes with high enough $\mathrm{S} / \mathrm{N}$, due to sensitivity limitations. With the advent of NASA's James Webb Space Telescope (JWST; see Kalirai 2018 for an up-todate review), MIR sensitivities will be increased by orders of magnitude compared to current ground-based facilities like VISIR on the VLT. As such, it will be possible in the future to measure subsecond variability of LMXBs at MIR and NIR wavelengths for the first time with JWST.

D.M.R. acknowledges the support of the NYU Abu Dhabi Research Enhancement Fund under grant RE124. T.M.B. acknowledges the financial contribution from the agreement ASIINAF n.2017-14-H.O. A.J.T. is supported by a Natural Sciences and Engineering Research Council of Canada (NSERC) PostGraduate Doctoral Scholarship (PGSD2-490318-2016). T.D.R. acknowledges support from the Netherlands Organisation for Scientific Research (NWO) Veni Fellowship, grant 639.041.646. J.C.A.M.-J. is the recipient of an Australian Research Council Future Fellowship (FT140101082). The research reported in this publication was supported by the Mohammed Bin Rashid Space Centre (MBRSC), Dubai, UAE, under grant ID 201701.SS. NYUAD. The Faulkes Telescope Project is an education partner of Las Cumbres Observatory. The Faulkes Telescopes are maintained and operated by LCO. This research has made use of the VizieR catalog access tool, CDS, Strasbourg, France. The original description of the VizieR service was published in Ochsenbein et al. (2000). F.C.Z. is supported by grant AYA2015-71042-P. S.C. acknowledges financial support from the UnivEarthS Labex program of Sorbonne Paris Cite (ANR-10-LABX-0023 and ANR11-IDEX-0005-02). J.M. acknowledges support from PNHE, the OCEVU Labex (ANR-11-LABX-0060), and the A*MIDEX project (ANR-11-IDEX-0001-02) funded by the "Investissements dAvenir" French government program managed by the ANR. G.R.S. and A.J.T. acknowledge funding from NSERC Discovery Grants. This work profited from discussions carried out during a meeting organized at the International Space Science Institute (ISSI) Beijing by T. Belloni and D. Bhattacharya. The SALT observations were obtained as part of the SALT Large Science Program on transients, 2016-2-LSP-001, which is in part supported by Polish participation in SALT funded by grant No. MNiSW DIR/WK/2016/07. D.B. acknowledges support from the National Research Foundation. This work is based on observations collected at the European Southern Observatory under ESO program ID 099.D-0884(A).

\section{ORCID iDs}

Maria Cristina Baglio (1) https://orcid.org/0000-00031285-4057

David M. Russell (1D https://orcid.org/0000-0002-3500-631X

Tomaso Belloni (i) https://orcid.org/0000-0001-9621-3796

Francesco Coti Zelati ib https://orcid.org/0000-0001-

7611-1581

Maria Díaz Trigo (1D https://orcid.org/0000-0001-7796-4279

Poshak Gandhi (iD https://orcid.org/0000-0003-3105-2615

Jeroen Homan (iD https://orcid.org/0000-0001-8371-2713

Karri I. I. Koljonen (iD https://orcid.org/0000-0002-9677-1533

Sera Markoff (ib https://orcid.org/0000-0001-9564-0876

James C. A. Miller-Jones (ib https://orcid.org/0000-00033124-2814

Thomas D. Russell (i) https://orcid.org/0000-0002-7930-2276

Greg R. Sivakoff (iD https://orcid.org/0000-0001-6682-916X

Vincenzo Testa (10 https://orcid.org/0000-0003-1033-1340

Alexandra J. Tetarenko (ib https://orcid.org/0000-00033906-4354

Mario E. van den Ancker (ib https://orcid.org/0000-00016992-3100

\section{References}

Anders, E., \& Grevesse, N. 1989, Geochim. Cosmochim. Acta, 53, 197 Blandford, R. D., \& Königl, A. 1979, ApJ, 232, 34

Buckley, D. A. H., Swart, G. P., \& Meiring, J. G. 2006, Proc. SPIE, 6267, $62670 Z$

Buxton, M. M., Bailyn, C. D., Capelo, H. L., et al. 2012, AJ, 143, 130

Cadolle Bel, M., Rodriguez, J., D’Avanzo, P., et al. 2011, A\&A, 534, A119

Casella, P., Maccarone, T. J., O'Brien, K., et al. 2010, MNRAS, 404, L21

Ceccobello, C., Cavecchi, Y., Heemskerk, M. H. M., et al. 2018, MNRAS, 473, 4417

Chaty, S., Dubus, G., \& Raichoor, A. 2011, A\&A, 529, A3

Corbel, S., \& Fender, R. P. 2002, ApJL, 573, L35

Corbel, S., Fender, R. P., Tomsick, J. A., Tzioumis, A. K., \& Tingay, S. 2004, ApJ, 617, 1272

Corbel, S., Aussel, H., Broderick, J. W., et al. 2013, MNRAS, 431, L107

Coriat, M., Corbel, S., Buxton, M. M., et al. 2009, MNRAS, 400, 123

Crawford, S. M., Still, M., Schellart, P., et al. 2010, Proc. SPIE, 7737, 773725

Diaz Trigo, M., Altamirano, D., Dincer, T., et al. 2018, A\&A, 616, A23

Díaz Trigo, M., Migliari, S., Miller-Jones, J. C. A., et al. 2017, A\&A, 600, A8

Dincer, T. 2017, ATel, 10716

Dinçer, T., Bailyn, C. D., Miller-Jones, J. C. A., Buxton, M., \& MacDonald, R. K. D. 2018, ApJ, 852, 4

Dinçer, T., Kalemci, E., Buxton, M. M., et al. 2012, ApJ, 753, 55 Dobrzycka, D., Momany, Y., Lundin, L., et al. 2012, Proc. SPIE, 8448, 84481P Drappeau, S., Malzac, J., Coriat, M., et al. 2017, MNRAS, 466, 4272 Drew, J. E., Gonzalez-Solares, E., Greimel, R., et al. 2014, MNRAS, 440, 2036 Drew, J. E., Gonzalez-Solares, E., Greimel, R., et al. 2016, yCat, 2341 Durant, M., Shahbaz, T., Gandhi, P., et al. 2011, MNRAS, 410, 2329

Falcke, H., Körding, E., \& Markoff, S. 2004, A\&A, 414, 895

Fender, R., Corbel, S., Tzioumis, T., et al. 1999, ApJL, 519, L165

Fender, R. P. 2001, MNRAS, 322, 31

Fender, R. P., Belloni, T. M., \& Gallo, E. 2004, MNRAS, 355, 1105

Fender, R. P., Homan, J., \& Belloni, T. M. 2009, MNRAS, 396, 1370

Foight, D. R., Güver, T., Özel, F., \& Slane, P. O. 2016, ApJ, 826, 66 Gandhi, P. 2009, ApJL, 697, L167

Gandhi, P., Bachetti, M., Dhillon, V. S., et al. 2017, NatAs, 1, 859

Gandhi, P., Blain, A. W., Russell, D. M., et al. 2011, ApJL, 740, L13

Gandhi, P., Dhillon, V. S., Durant, M., et al. 2010, MNRAS, 407, 2166

Gandhi, P., Littlefair, S. P., Hardy, L. K., et al. 2016, MNRAS, 459, 554

Heinz, S., \& Sunyaev, R. A. 2003, MNRAS, 343, L59

Homan, J., Buxton, M., Markoff, S., et al. 2005, ApJ, 624, 295

Hynes, R. I., Robinson, E. L., Pearson, K. J., et al. 2006, ApJ, 651, 401

Jain, R. K., Bailyn, C. D., Orosz, J. A., McClintock, J. E., \& Remillard, R. A. 2001, ApJL, 554, L181

Kalamkar, M., Casella, P., Uttley, P., et al. 2016, MNRAS, 460, 3284

Kalberla, P. M. W., Burton, W. B., Hartmann, D., et al. 2005, A\&A, 440, 775

Kalemci, E., Dinçer, T., Tomsick, J. A., et al. 2013, ApJ, 779, 95

Kalirai, J. 2018, ConPh, 59, 251 
Kennea, J. A. 2017, ATel, 10731

Kennea, J. A., Evans, P. A., Beardmore, A. P., et al. 2017, ATel, 10700

Koljonen, K. I. I., Russell, D. M., Fernández-Ontiveros, J. A., et al. 2015, ApJ, 814, 139

Lagage, P. O., Pel, J. W., Authier, M., et al. 2004, Msngr, 117, 12

Lasso-Cabrera, N. M., \& Eikenberry, S. S. 2013, ApJ, 775, 82

Lewis, F., Roche, P., Russell, D. M., \& Fender, R. P. 2008, in AIP Conf. Ser.1010, A Population Explosion: The Nature Evolution of X-ray Binaries in Diverse Environments, ed. R. M. Bandyopadhyay et al. (Melville, NY: AIP), 204

Malzac, J. 2013, MNRAS, 429, L20

Malzac, J. 2014, MNRAS, 443, 299

Meier, D. L. 2001, ApJL, 548, L9

Merloni, A., Heinz, S., \& di Matteo, T. 2003, MNRAS, 345, 1057

Migliari, S., Tomsick, J. A., Miller-Jones, J. C. A., et al. 2010, ApJ, 710, 117

Motta, S. E., Rouco Escorial, A., Kuulkers, E., Muñoz-Darias, T., \& Sanna, A. 2017, MNRAS, 468, 2311

Nakahira, S., Negoro, H., Mihara, T., et al. 2017, ATel, 10729

Negoro, H., Kawase, T., Sugizaki, M., et al. 2017a, ATel, 10708

Negoro, H., Ishikawa, M., Ueno, S., et al. 2017b, ATel, 10699

Oates, S. R., Motta, S., Beardmore, A. P., et al. 2018, arXiv:1809.03237

Ochsenbein, F., Bauer, P., \& Marcout, J. 2000, A\&AS, 143, 23

O'Donoghue, D., Buckley, D. A. H., Balona, L. A., et al. 2006, MNRAS, 372, 151

Palmer, D. M., Krimm, H. A. \& Swift/BAT Team 2017, ATel, 10733

Plotkin, R. M., Gallo, E., \& Jonker, P. G. 2013, ApJ, 773, 59

Plotkin, R. M., Gallo, E., Jonker, P. G., et al. 2016, MNRAS, 456, 2707

Ponti, G., Fender, R. P., Begelman, M. C., et al. 2012, MNRAS, 422, 11

Rahoui, F., Lee, J. C., Heinz, S., et al. 2011, ApJ, 736, 63

Russell, D. M., Fender, R. P., Hynes, R. I., et al. 2006, MNRAS, 371, 1334

Russell, D. M., Maitra, D., Dunn, R. J. H., \& Markoff, S. 2010, MNRAS, 405,1759
Russell, D. M., Miller-Jones, J. C. A., Maccarone, T. J., et al. 2011, ApJL, 739, L19

Russell, D. M., Qasim, A. A., Bernardini, F., et al. 2018, ApJ, 852, 90

Russell, D. M., Russell, T. D., Miller-Jones, J. C. A., et al. 2013a, ApJL, 768, L35

Russell, D. M., Markoff, S., Casella, P., et al. 2013b, MNRAS, 429, 815

Russell, T. D., Altamirano, D., Tetarenko, A. J., et al. 2017a, ATel, 10899

Russell, T. D., Miller-Jones, J. C. A., Sivakoff, G. R., Tetarenko, A. J. \& Jacpot Xrb Collaboration 2017b, ATel, 10711

Russell, T. D., Soria, R., Miller-Jones, J. C. A., et al. 2014, MNRAS, 439, 1390

Scaringi, S. e. a. 2017, ATel, 10702

Schlafly, E. F., \& Finkbeiner, D. P. 2011, ApJ, 737, 103

Shahbaz, T., Russell, D. M., Covino, S., et al. 2016, MNRAS, 463, 1822

Shang, J.-R., Debnath, D., Chatterjee, D., et al. 2018, arXiv:1806.07147

Shidatsu, M., Nakahira, S., Negoro, H., et al. 2017a, ATel, 11020

Shidatsu, M., Nakahira, S., Negoro, H., et al. 2017b, ATel, 10761

Skrutskie, M. F., Cutri, R. M., Stiening, R., et al. 2006, AJ, 131, 1163

Smith, J. A., Allam, S. S., Tucker, D. L., et al. 2005, BAAS, 207, 131.11

Tananbaum, H., Gursky, H., Kellogg, E., Giacconi, R., \& Jones, C. 1972, ApJL, 177, L5

Tao, L., Chen, Y., Güngör, C., et al. 2018, MNRAS, 480, 4443

Tetarenko, A. J., Russell, T. D., Miller-Jones, J. C. A., Sivakoff, G. R. \& Jacpot Xrb Collaboration 2017, ATel, 10745

Tetarenko, A. J., Sivakoff, G. R., Miller-Jones, J. C. A., et al. 2018, MNRAS, submitted (arXiv:1810.05709)

Vadawale, S. V., Rao, A. R., Naik, S., et al. 2003, ApJ, 597, 1023

van Boekel, R., Juhász, A., Henning, T., et al. 2010, A\&A, 517, A16

Vaughan, S., Edelson, R., Warwick, R. S., \& Uttley, P. 2003, MNRAS, 345, 1271

Vincentelli, F. M., Casella, P., Maccarone, T. J., et al. 2018, MNRAS, 477, 4524

Weingartner, J. C., \& Draine, B. T. 2001, ApJ, 548, 296

Wilms, J., Allen, A., \& McCray, R. 2000, ApJ, 542, 914 